\title{
Further examination of the thermodynamic modification of the inflow layer of tropical cyclones by vertical wind shear
}

\author{
M. Riemer ${ }^{1}$, M. T. Montgomery ${ }^{2,3}$, and M. E. Nicholls ${ }^{4}$ \\ ${ }^{1}$ Institut für Physik der Atmosphäre, Johannes Gutenberg-Universität, Mainz, Germany \\ ${ }^{2}$ Department of Meteorology, Naval Postgraduate School, Monterey, CA, USA \\ ${ }^{3}$ NOAA's Hurricane Research Division, Miami, FL, USA \\ ${ }^{4}$ University of Colorado, Cooperative Institute for Research in Environmental Sciences, Boulder, CO, USA \\ Correspondence to: M. Riemer (mriemer@uni-mainz.de)
}

Received: 15 October 2011 - Published in Atmos. Chem. Phys. Discuss.: 7 March 2012

Revised: 20 November 2012 - Accepted: 1 January 2013 - Published: 11 January 2013

\begin{abstract}
Recent work has developed a new framework for the impact of vertical wind shear on the intensity evolution of tropical cyclones. A focus of this framework is on the frustration of the tropical cyclone's power machine by shearinduced, persistent downdrafts that flush relatively cool and dry (lower equivalent potential temperature, $\theta_{\mathrm{e}}$ ) air into the storm's inflow layer. These previous results have been based on idealised numerical experiments for which we have deliberately chosen a simple set of physical parameterisations. Before efforts are undertaken to test the proposed framework with real atmospheric data, we assess here the robustness of our previous results in a more realistic and representative experimental setup by surveying and diagnosing five additional numerical experiments. The modifications of the experimental setup comprise the values of the exchange coefficients of surface heat and momentum fluxes, the inclusion of experiments with ice microphysics, and the consideration of weaker, but still mature tropical cyclones.

In all experiments, the depression of the inflow layer $\theta_{\mathrm{e}}$ values is significant and all tropical cyclones exhibit the same general structural changes when interacting with the imposed vertical wind shear. Tropical cyclones in which strong downdrafts occur more frequently exhibit a more pronounced depression of inflow layer $\theta_{\mathrm{e}}$ outside of the eyewall in our experiments. The magnitude of the $\theta_{\mathrm{e}}$ depression underneath the eyewall early after shear is imposed in our experiments correlates well with the magnitude of the ensuing weakening of the respective tropical cyclone. Based on the evidence presented, it is concluded that the newly proposed framework is a robust description of intensity modification in our suite of experiments.
\end{abstract}

\section{Introduction}

\subsection{A new framework for intensity modification of tropical cyclones in vertical wind shear}

The intensity evolution of tropical cyclones (TCs) embedded in an environmental flow with vertical wind shear poses an important forecast problem. To date, the physical processes that govern this intensity evolution are not well understood. In a recent publication (Riemer et al., 2010, referred to as RMN10 hereafter) a new framework for the intensity modification of TCs in vertical wind shear was proposed. While previous hypotheses have focused almost exclusively on processes above the boundary layer, RMN10 showed that vertical wind shear has a significant impact on the thermodynamic properties of the TC's inflow layer ${ }^{1}$. Strong and persistent, shear-induced downdrafts flush the inflow layer with low moist-entropy (or equivalent potential temperature, $\theta_{\mathrm{e}}$ ) air - "anti-fuel" for the TC power machine. The replenishment of this air by surface heat fluxes is not complete while the air spirals towards the storm centre leading to a reduction of $\theta_{\mathrm{e}}$ values within the inner-core updrafts in the azimuthal mean. When viewed from the simplified perspective of an idealised Carnot cycle, a decrease in the potential intensity of the TC can be expected (Tang and Emanuel, 2010).

\footnotetext{
${ }^{1}$ In the current study, the term "inflow layer" will refer to the layer of strong inflow associated with surface friction in the lowest $1-1.5 \mathrm{~km}$ where the departure from gradient wind balance in the radial direction is generally a maximum (e.g., Bui et al. (2009), their Figs. 5 and 6 and accompanying discussion).
} 
The downdrafts that flush the inflow layer with low- $\theta_{\mathrm{e}}$ air are associated with a quasi-stationary, azimuthal wavenumber 1, convective asymmetry outside of the eyewall. This convective asymmetry is reminiscent of the "stationary band complex" (Willoughby et al., 1984) and for this reason will be referred to as the SBC hereafter. The SBC and the associated downdraft pattern extends outwards to approximately $150-180 \mathrm{~km}$. On account of the swirling winds, the updrafts within the SBC rise along a helical path. A simple kinematic model (Riemer and Montgomery, 2011) suggests that, at low levels above the inflow layer, environmental low- $\theta_{\mathrm{e}}$ air approaches the storm closely underneath the helical updrafts in the downshear and downshear-left quadrants ${ }^{2}$. Evaporative cooling of precipitation falling out of the SBC, and into this unsaturated air, most likely drives the strong and persistent, vortex-scale downdrafts that cool and dry the TC's inflow layer.

In RMN10, evidence was presented that the formation of the SBC is connected to the tilt ${ }^{3}$ of the TC vortex. When subject to vertical wind shear, the tilt evolution of the TC vortex was shown to be governed to zero order by asymmetricbalance dynamics. Vortex tilt can thus be described in terms of a standing vortex-Rossby-wave (VRW) pattern with azimuthal and vertical wave-number 1 structure. Even though the tilt of the inner core is only $10-20 \mathrm{~km}$, the VRW pattern associated with the tilt of the outer vortex extends outwards to radii of $150-200 \mathrm{~km}$ (Fig. 15a in RMN10) ${ }^{4}$. After vertical wind shear is suddenly imposed, the TC settles quickly (approx. within $3 \mathrm{~h}$ ) into a quasi-equilibrium tilt direction that is to the downshear-left. Thus, the positive vorticity anomaly associated with the standing wave-number 1 VRW pattern is found downshear-left at upper levels and downshear-right at low levels (Fig. 16 in RMN10). Frictional convergence associated with this positive low-level vorticity anomaly then contributes to the forcing of the asymmetric convection within the SBC. The foregoing results suggest an important connection between the asymmetric-balance dynamics governing the tilt evolution of the vortex subject to vertical shear and the thermodynamic impact on the inflow layer.

The findings of RMN10 were derived from idealised numerical experiments following the setup of Bender (1997) and Frank and Ritchie (2001). In this experimental setup, a uni-directional vertical wind shear profile is imposed suddenly on a mature model TC. In an attempt to min-

\footnotetext{
${ }^{2}$ In Riemer and Montgomery (2011), the distribution of $\theta_{\mathrm{e}}$ was analysed at $2 \mathrm{~km}$ height.

${ }^{3}$ In RMN10, as well as in the current study, the tilt is defined as the vector difference between the location of the vorticity centroids at $10 \mathrm{~km}$ and $1 \mathrm{~km}$ height.

${ }^{4} \mathrm{~A}$ considerable tilt of the outer parts of TC-like vortices with small tilt of the inner core has been noted previously by other authors also (see e.g. Fig. 6a in Jones, 1995; Fig. 10 in Reasor et al., 2004).
}

imise complexity and to understand the fundamental processes, RMN10 deliberately chose a simple set of model parameterisations. As is the nature of results derived from such an idealised approach, uncertainties exist whether the newly proposed pathway operates in the real atmosphere. Although some preliminary observational support was offered in RMN10 a more comprehensive test of the new hypothesis with atmospheric data awaits future studies. Before undertaking such efforts we survey and diagnose here five additional numerical experiments with some modifications of the experimental setup to assess the robustness of RMN10's results.

\subsection{Purpose of additional experiments}

The experimental setup in RMN10 features a simplified cloud microphysics scheme, a likely overestimation of the surface exchange coefficients of momentum and enthalpy at high wind speed, and a very high TC intensity at the time when shear is imposed, representative for a minority of TCs in the real atmosphere only. The particular relevance of these features for RMN10's framework is discussed in more detail below. One goal of this study is to assess the robustness of RMN10's results in a more realistic and representative experimental setup.

Several environmental factors likely play a role for the evolution of TCs in vertical wind shear also. Besides the obvious importance of the shear magnitude, such factors include the vertical profiles of environmental wind speed and direction (Zeng et al., 2010; Wang, 2012), and the environmental moisture and temperature profiles (cf. discussion in Riemer and Montgomery (2011)). Careful examination of the importance of these environmental profiles is beyond the scope of this study but constitutes an important topic for future research ${ }^{5}$.

The numerical experiments in RMN10 employed a microphysics scheme that considers "warm cloud" processes associated with the collision-coalescence process only (hereafter termed "warm rain"). The current study considers experiments with ice microphysics also. Adding ice processes introduces the latent heat of fusion/ sublimation, new classes of hydrometeors with various fall speeds, and additional pathways to form precipitation. Details of the ice microphysics scheme employed in this study are given in Sect. 2.2. Arguably, the most significant of the above processes is the additional conversion of latent heat associated with the freezing and melting of ice. Ice microphysics produce stronger updrafts than a warm-rain scheme due to the additional latent heat release associated with freezing. In addition, the inclusion of precipitation in the form of ice may significantly enhance the formation and intensity of downdrafts (Srivastava, 1987).

\footnotetext{
${ }^{5} \mathrm{~A}$ brief discussion of the potential consequences of the environmental wind profile can be found in the authors' response to the anonymous reviewer (item 1 and 2 ) on the ACPD webpage.
} 
Previous studies have shown that the inclusion of ice microphysics in numerical models may alter the radial profile of the swirling winds of TCs as compared to a warm-rain experiment (e.g., Lord et al., 1984; Fovell et al., 2009). In general, it is not well understood how these differences arise ${ }^{6}$. Lord et al. have noted, albeit in an axisymmetric model framework, that their experiment with ice microphysics exhibits more mesoscale convective features than their experiment with warm rain. Fovell et al. have proposed a mechanism for the change in the radial profile of the swirling winds that is based on the sedimentation of ice particles from the outflow anvil, the subsequent sublimentation of these ice particles, and radiative feedback. In our suite of experiments, however, radiative processes are neglected. Nevertheless, potentially significant differences in the TC-vortex structure between the warm-rain and the ice microphysics experiments do arise, indicating that the mechanism suggested by Fovell et al. is not of primary importance. The potentially important differences in the TC-vortex structure and their implication for the interaction with vertical wind shear will be discussed in some detail in Sect. 3.1.

Recent studies have highlighted the importance of the representation of the planetary boundary layer in numerical models for vortex intensification and structure changes of simulated storms (Braun and Tao, 2000; Smith and Thomsen, 2010; Montgomery et al., 2010; Smith et al., 2012). The exchange coefficients for enthalpy, $C_{K}$, and momentum, $C_{D}$, play a most prominent role. They govern the energy extraction from the ocean surface and the dissipation of kinetic energy in the frictional boundary layer, respectively. In the framework of RMN10, the values of $C_{K}$ and $C_{D}$ can be expected to play an important role in the replenishment of the depressed boundary layer $\theta_{\mathrm{e}}$ air while spiralling towards the inner-core updrafts. The replenishment rate is proportional ${ }^{7}$ to $C_{K}$. The strength of the inflow within the frictional boundary layer is determined in part by $C_{D}$. Smaller values of $C_{D}$ may lead to less radial inflow and thus to an increase of the time that the air parcels spend under the influence of surface fluxes while spiralling inwards. Assuming that $C_{K}$ is not changed, and that the wind dependence of the fluxes is dominated by the tangential wind speed, the replenishment of depressed inflow layer $\theta_{\mathrm{e}}$ values is then more complete than for larger values of $C_{D}$. Based on these considerations, one would expect that the shear-induced flushing of the inflow layer with low- $\theta_{\mathrm{e}}$ air has a more pronounced impact on TC intensity for smaller values of $C_{K}$ and larger values of

\footnotetext{
${ }^{6}$ In his review of this study, Roger Smith has proposed that a first-order explanation of how these differences arise could be given in terms of the conventional (balanced) model of vortex spin-up, noting that the inclusion of ice microphysics substantially modifies the diabatic heating rate. We are not aware, however, of published studies that elaborate on this idea.

${ }^{7}$ The replenishment rate is proportional to the air-sea thermodynamic disequilibrium also.
}

$C_{D}$. The interpretation of the importance of $C_{K}$ and $C_{D}$ in an axisymmetric theory for a TC in vertical wind shear by Tang and Emanuel (2010) is consistent with the foregoing considerations.

Considerable uncertainties exist about the values of $C_{K}$ and $C_{D}$ at the high wind speeds occurring in TCs. Deacon's formula for $C_{D}$ was employed in RMN10 and the ratio of $C_{K} / C_{D}$ was set to unity. Recent results derived from observational data (Black et al., 2007; Zhang et al., 2008) strongly indicate that Deacon's formula overestimates $C_{D}$ at high wind speeds and that $C_{K} / C_{D}$ is considerably less than unity. The observational data exhibit considerable scatter and are limited to wind speeds of marginal hurricane strength ${ }^{8}$. To address the likely overestimation of $C_{K}$ and $C_{K} / C_{D}$ in RMN10, we employ in this study values of $C_{K}$ and $C_{K} / C_{D}$ that are consistent with, but rather on the lower end of the range of values observed by Black et al. and Zhang et al. Further details are provided in Sect. 2.3.

The TCs considered in RMN10 were intense and rapidly intensifying at the time when the vertical wind shear profile was imposed. In the current study we will consider, inter alia, weaker, but still mature TCs. Finally, our additional experiments now exhibit various rates of intensification just before vertical wind shear is imposed. We did not, however, find a sensitivity of the subsequent TC evolution in vertical shear to this preceding intensification rate in our experiments. Thus, the various rates of intensification just before vertical wind shear is imposed will not be considered hereafter.

The next section provides an overview of the numerical model employed and the additional experiments performed, an expanded description of the ice microphysics scheme, and the values for the surface exchange coefficients. In Sect. 3 we compare the evolution of the quintessential ingredients of RMN10's newly proposed framework in the different numerical experiments. One experiment with ice microphysics is examined in further detail in Sect. 4, including an estimate of the spindown timescale of the simulated TC-vortex based on simple axisymmetric theory. Section 5 contains our conclusions.

\section{Model setup and additional experiments}

\subsection{A brief model description}

For the numerical experiments we employ the Regional Atmospheric Modeling System (RAMS), developed at Colorado State University (Pielke et al., 1992; Cotton et al., 2003). The RAMS is a three-dimensional, nonhydrostatic numerical modeling system comprising timedependent equations for velocity, non-dimensional pressure

\footnotetext{
${ }^{8}$ Some significant strides towards determining these coefficients at major hurricane wind conditions have been made recently by Bell et al. (2012).
} 
perturbation, ice-liquid water potential temperature (Tripoli and Cotton, 1981), and mixing ratios of total water and hydrometeors.

The experiments are carried out on an f-plane at $15^{\circ} \mathrm{N}$ and with a horizontal grid spacing of $5 \mathrm{~km}$. The model does not include ocean feedback. The time-invariant sea surface temperature is set to $28.5^{\circ} \mathrm{C}$. A standard first-order turbulence scheme developed by Smagorinsky (1963) is used for subgrid-scale mixing, with modifications by Lilly (1962) and Hill (1974) that enhance diffusion in unstable conditions and reduce diffusion in stable conditions. For simplicity, radiative processes are neglected. The bulk aerodynamic formulas are used to calculate momentum fluxes and sensible and latent heat fluxes from the sea surface. The values of the exchange coefficients are given in Sect. 2.3. We employ either a warmrain microphysics scheme based on that of Kessler (1969) or the RAMS standard ice microphysics scheme (Sect. 2.2). The mixing ratio equations for all prognosed hydrometeors include advection by the three-dimensional flow. A more comprehensive description of RAMS, the surface interaction parameterisation, and the warm-rain scheme is given in RMN10.

\subsection{Ice microphysics in RAMS}

The RAMS ice microphysics scheme has eight water categories: vapour, cloud droplets, rain, pristine ice, snow, aggregates, graupel and hail (Walko et al., 1995; Meyers et al., 1997). The liquid phase categories, cloud droplets and rain, may be supercooled. Pristine ice, snow and aggregates are assumed to be completely frozen, whereas graupel and hail are mixed phase categories, capable of consisting of ice only, or a mixture of ice and liquid. All hydrometeor categories except cloud droplets are assumed large enough to fall. Cloud droplets and pristine ice are the only categories to nucleate from vapour. All other categories form from existing hydrometeors, but once formed may grow further by vapour deposition. Pristine ice crystals form from heterogeneous nucleation, homogeneous nucleation, and secondary production that involves splintering of riming crystals. The snow category is defined in RAMS as relatively large ice crystals, which have grown from the pristine ice category due to vapour deposition and may continue to grow by riming. Aggregates are defined as ice particles that have formed by collision and coalescence of pristine ice, snow and/or aggregates. Snow and aggregates retain their identity with moderate amounts of riming, but convert to graupel if the amount of riming is large. In addition to forming by heavy riming, graupel can also form by partial melting of snow or aggregates. Graupel is allowed to carry up to half its mass in liquid. If the percentage becomes larger, by either melting, riming or collection by rain, a graupel particle is re-categorised as hail. Hail is formed by freezing of rain drops or by riming or partial melting of graupel. Hail is allowed to be a large fraction liquid and converts to rain when completely liquid.
The hydrometeor size distribution in RAMS is represented by a generalised Gamma function. A user specified shape parameter controls the width of the distribution. Also, a scaling diameter is often specified for a one-moment scheme that is related to the mean diameter of the distribution, with the particular relation depending on the chosen spectral parameters. For this study a one-moment scheme is employed for all categories except cloud droplets and pristine ice. A scaling diameter is specified and the number concentration is diagnosed using the predicted mixing ratio of the hydrometeor scheme.

A heat budget equation is formulated for rain, graupel, and hail hydrometeors. This allows the temperature of a hydrometeor to differ from that of the surrounding ambient air temperature due to latent heat release or absorption in the hydrometeor, and sensible heating by collisions with other hydrometeors. The temperature of a hydrometeor often differs substantially from that of the air, which can significantly influence the rates of heat and vapour diffusion, as well as the amount of sensible heat transfer that occurs in coalescence of hydrometeors. Collision and coalescence of hydrometeors is governed by the stochastic equation derived by Verlinde et al. (1990).

Gravitational settling of hydrometeors causes them to fall relative to air. Terminal velocities increase as a power of the droplet diameter, and are based on empirical data. Pristine ice has very small fall speeds. Snow crystals fall faster than pristine ice, but still relatively slowly. Aggregates are large crystals that have moderate fall speeds. Graupel and hail have high fall speeds. Sedimentation in RAMS uses a Lagrangian scheme to transport the mixing ratio from any given grid cell to a lower height in the vertical column, so that a proportion is transferred to the lower adjacent grid cell.

\subsection{Modification of surface exchange coefficients}

Deacon's formula for the dependence of the drag exchange coefficient $C_{D}$ on wind speed is given by

$C_{D}=1.1 \times 10^{-3}+4 \times 10^{-5}|V|_{1}$,

with $|V|_{1}$ being the wind speed in $\mathrm{m} \mathrm{s}^{-1}$ at the lowest model level (here: $49 \mathrm{~m}$ ). In RMN10 we used Deacon's formula together with $C_{K}=C_{D}$. For reference, this formula furnishes values of the exchange coefficients of $1.5 \times 10^{-3}, 2.1 \times 10^{-3}$, and $3.1 \times 10^{-3}$ for $10 \mathrm{~m} \mathrm{~s}^{-1}, 25 \mathrm{~m} \mathrm{~s}^{-1}$, and $50 \mathrm{~m} \mathrm{~s}^{-1}$, respectively.

Zhang et al. (2008) found that "The exchange coefficient of enthalpy flux shows no significant dependence on wind speed up to hurricane force with a value of $1.16 \times 10^{-3}$." and that "The average ratio of the $C_{K} / C_{D}$ values is $0.63, \ldots$ ". We therefore use these values and set

$C_{K}=1.16 \times 10^{-3}=\mathrm{constant}$

and

$C_{K} / C_{D}=0.63$. 
Table 1. Overview of the experiments that are considered in this study. The experiments $\mathrm{RMN}_{68}$ and ${ }_{10} \mathrm{RMN}_{68}$ are described in detail in RMN10. In column $C_{K}$, "Deacon" denotes that Deacon's formula (see Sect. 2.3) is used to determine the exchange coefficients. The last column gives the TC's intensification rate just before vertical shear is imposed.

\begin{tabular}{lcccccc}
\hline Experiment & $\begin{array}{c}\text { "initial" } \\
\text { intensity }\left[\mathrm{m} \mathrm{s}^{-1}\right]\end{array}$ & $C_{K}$ & $C_{K} / C_{D}$ & microphysics & $\begin{array}{c}\text { shear } \\
{\left[\mathrm{m} \mathrm{s}^{-1} / 12 \mathrm{~km}\right]}\end{array}$ & $\begin{array}{c}\text { preceding int. } \\
\text { rate }\left[\mathrm{m} \mathrm{s}^{-1} / \mathrm{h}\right]\end{array}$ \\
\hline $\mathrm{RMN}_{68}$ & 68 & Deacon & 1 & warm & 15 & 1.8 \\
$1_{10} \mathrm{RMN}_{68}$ & 68 & Deacon & 1 & warm & 10 & 1.8 \\
$\mathrm{RMN}_{54}$ & 54 & Deacon & 1 & warm & 15 & 0.0 \\
CBLAST $_{68}$ & 68 & $1.16 \times 10^{-3}$ & 0.65 & warm & 15 & 1.1 \\
$\mathrm{CBLAST}_{54}$ & 54 & $1.16 \times 10^{-3}$ & 0.65 & warm & 15 & 0.0 \\
$\mathrm{ICE}_{68}$ & 68 & Deacon & 1 & ice & 15 & 2.5 \\
$10 \mathrm{ICE}_{68}$ & 68 & Deacon & 1 & ice & 10 & 2.5 \\
\hline
\end{tabular}

Experiments that employ these values are referred to as CBLAST below. Note that Deacon's formula and $C_{K} / C_{D}=$ 1 furnishes values of $C_{K}$ and $C_{D}$ that, for $|V|_{1}=50 \mathrm{~m} \mathrm{~s}^{-1}$, are approx. 2.7 and 4.2 times higher, respectively, than the corresponding CBLAST values.

\subsection{Summary of experiments}

A summary of the experiments examined in this study is given in Table 1 . We refer to experiments that employ the same parameterisations as in RMN10, i.e. warm rain and Deacon's formula for the exchange coefficients (with $C_{K} / C_{D}=1$ ), as RMN. The CBLAST experiments employ warm rain also. Experiments that include ice microphysics are referred to as ICE. ICE experiments use Deacon's formula with $C_{K} / C_{D}=1$. Besides the modifications that are mentioned explicitly, the experimental setup is the same as in RMN10.

The shear profile employed in this study has a sine structure in the vertical with zero winds at the surface and maximum easterly winds at $12 \mathrm{~km}$ and above. We will follow the convention of the TC community to define vertical wind shear as the vector difference between the winds at upper and lower levels. Here, we use the vector difference between $12 \mathrm{~km}$ and the surface. The shear magnitude is thus given in $\mathrm{m} \mathrm{s}^{-1}$. Unless otherwise noted, we will consider TCs in vertical wind shear with a magnitude of $15 \mathrm{~m} \mathrm{~s}^{-1}$. The shear profile is imposed at a high intensity ${ }^{9}$ of $68 \mathrm{~m} \mathrm{~s}^{-1}$, after the TCs have spun up in quiescent environment. These experiments are referred to as $\mathrm{RMN}_{68}, \mathrm{CBLAST}_{68}$, and $\mathrm{ICE}_{68}$, respectively. The intensity at the time when shear is imposed will be referred to as "initial intensity" below. The conventions used in this study follow those in RMN10. Experiment $\mathrm{RMN}_{68}$ is the "15mps" case of RMN10 and constitutes our reference experiment.

We have performed experiments with a weaker initial intensity of $54 \mathrm{~m} \mathrm{~s}^{-1}$ also, referred to as $\mathrm{RMN}_{54}$ and

\footnotetext{
${ }^{9}$ Our storm intensity metric is the maximum azimuthallyaveraged tangential wind speed at $1 \mathrm{~km}$ height.
}

CBLAST $_{54}$. These experiments use the same spinup runs as the respective experiments with initial intensity of $68 \mathrm{~m} \mathrm{~s}^{-1}$, but with vertical shear imposed earlier during the intensification. Furthermore, an ICE experiment has been performed with $10 \mathrm{~m} \mathrm{~s}^{-1}$ vertical shear $\left({ }_{10} \mathrm{ICE}_{68}\right)$ which will be compared briefly to the "10mps" case of RMN10 (here: $\left.{ }_{10} \mathrm{RMN}_{68}\right)$.

\section{Assessing the robustness of the new framework}

We shall focus on the key features of RMN10's new framework for intensity modification in vertical wind shear: the vortex tilt, the occurrence and location of the SBC, the flushing of the inflow layer with low- $\theta_{\mathrm{e}}$ air, the ensuing depression of inflow layer $\theta_{\mathrm{e}}$, and the associated weakening of the TC. The general characteristics of these key features will be found to be common in all experiments.

As can be expected, differences arise in the specific characteristics of these key features. For some of these differences it is very difficult to establish the causal link to the changes in the experimental setup. Most prominently, differences between the evolution of the TCs in $\mathrm{RMN}_{68}$ and $\mathrm{ICE}_{68}$ are partly due to the enhancement of downdrafts by ice microphysics (Sect. 3.7), but very likely due also to changes in the radial profile of the swirling winds at the time when vertical wind shear is imposed. Disentangling the individual contributions requires a more complete understanding of the processes at work, and extended numerical experimentation and diagnostic analyses, preferably in an ensemble-based framework. A more comprehensive examination of such differences is thus deferred to future research.

\subsection{Importance of the radial profile of the swirling winds}

\subsubsection{Impact on the resiliency of TC-like vortices}

It is generally believed that the presence of vertical wind shear increases the interaction between a TC and dry environmental air (Riemer and Montgomery, 2011, and references 
therein). Based on a simple kinematic model, Riemer and Montgomery (2011) argue that a TC with a broad radial profile of the swirling winds should have a stronger ability to isolate itself from the detrimental interaction with dry environmental air than a smaller-scale TC. Furthermore, the radial profile of the swirling winds has a profound impact on the dynamic resiliency of TC-like vortices, i.e. the ability to maintain vertical coherence in the presence of differential advection by the vertical shear flow (e.g., Jones, 1995; Reasor et al., 2004). These studies investigated the resiliency of initially barotropic, dry vortices and are reviewed briefly below.

Jones (1995) examined vortex evolution in terms of the formation and subsequent interaction of potential vorticity (PV) anomalies. PV anomalies were defined as deviations from the azimuthal mean. Due to the differential advection by the vertical shear flow, PV anomalies representing the tilt of the vortex form at lower and upper levels. These anomalies mutually interact through the vertical penetration of their "induced" quasi-horizontal circulations, causing the vortex to precess. This precession helps the vortex to counteract the differential advection by the vertical wind shear. The vertical interaction of these PV anomalies depends on the Rossby penetration depth. The penetration depth increases with the strength of the background rotation and with the horizontal scale of the interacting anomalies ${ }^{10}$. Jones noted that it is not entirely clear how to quantify the penetration depth for a tilted TC-like vortex but concluded that intense and broad vortices are more resilient than their weaker, smaller-scale counterparts.

Reasor et al. (2004) re-visited the problem of vortex resiliency and re-alignment using VRW concepts and models. Their results are in qualitative agreement with Jones's finding that vortex (and environment) parameters that increase the penetration depth improve the resistence of a vortex to tilting by an imposed vertical shear flow. In addition, Reasor et al. demonstrated that vortex precession is not the only element in the resiliency of TC-like vortices. An inviscid damping mechanism of the vortex-tilt mode exists, associated with PV mixing at the critical radius of the near-discrete VRW that represents the vortex tilt. The critical radius is typically located in the "skirt" region of the vortex, i.e. in the region of small PV values outside of the high PV values in the inner core (approx. outside $50-70 \mathrm{~km}$ in Fig. 1a). The inviscid damping mechanism depends on the radial gradient of the skirt PV at the critical radius (Schecter and Montgomery, 2003). Inviscid damping, and thus re-alignment of the vortex, occurs when the radial PV gradient in this region is negative. The damping rate increases with the magnitude of the gradient. For a positive skirt-PV gradient, a tilt instability may occur. The results of Reasor et al. clearly demonstrate that vortex resiliency may crucially depend on the radial profile of the swirling winds.

\footnotetext{
${ }^{10}$ The penetration depth increases also with a decrease in static stability.
}
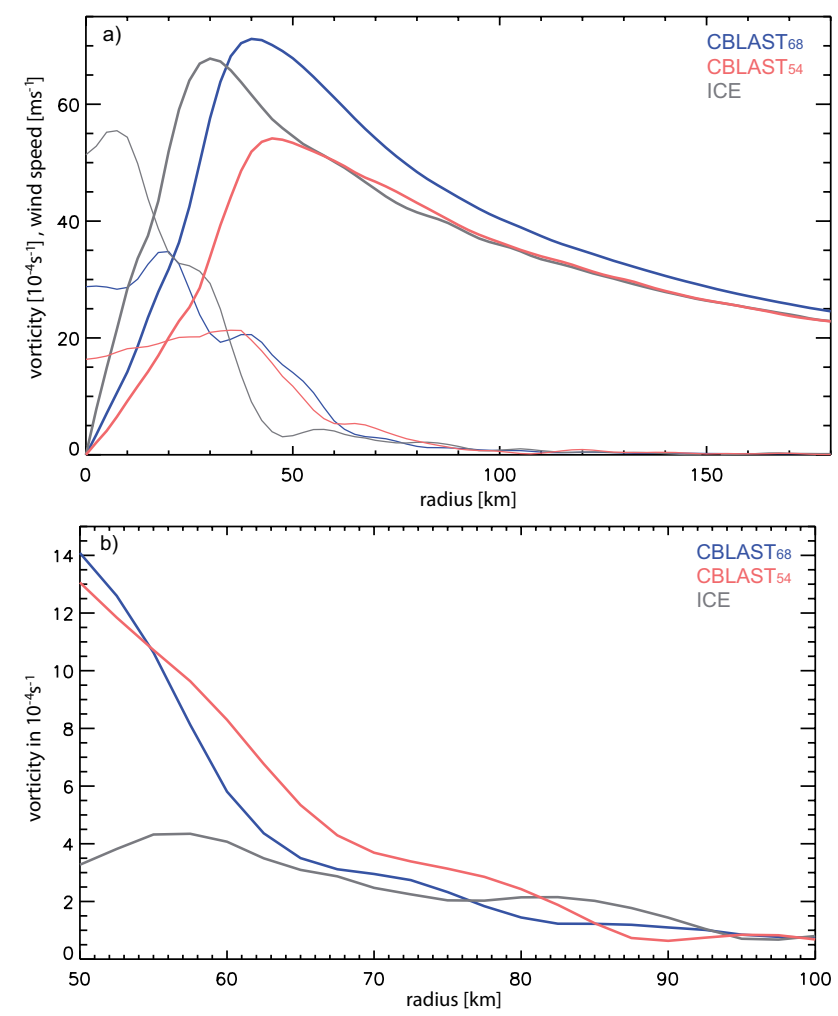

Fig. 1. (a) Radial profiles of azimuthal mean vorticity at $2 \mathrm{~km}$ (thin) and azimuthal mean tangential wind at the radius of maximum winds at $1 \mathrm{~km}$ height (thick) at the time when vertical wind shear is imposed in $\mathrm{CBLAST}_{54}$ (red), $\mathrm{CBLAST}_{68}$ (blue), and $\mathrm{ICE}_{68}$ (grey), respectively. (b) Radial vorticity profiles as in (a) but zoomed in on the "skirt" region between $50 \mathrm{~km}$ and $100 \mathrm{~km}$.

The radial profile of the swirling winds has been confirmed to play a role in the intensity evolution of vertically sheared TCs in idealised, moist numerical experiments (Wong and Chan, 2004). In these experiments the weakening of smallerscale TCs is found to be more pronounced than that of their broader-scale counterparts. Wong and Chan based their interpretation of this difference in the intensity evolution on the results of Jones (1995) derived from dry TC-like vortices. To us, however, it is not clear what the underlying processes are that govern the intensity evolution in Wong and Chan's experiments. In particular, the role of moist processes and/or the associated secondary circulation remains unclear.

In the real atmosphere, an indication of a higher susceptibility of smaller-scale TCs to vertical shear has been found by DeMaria (1996) based on a regression analysis.

\subsubsection{Diagnosed radial vortex structure in our experiments}

Because the radial profile of the swirling winds may play a prominent role in the evolution of TCs in vertical wind shear, we begin the examination of our experiments with the 

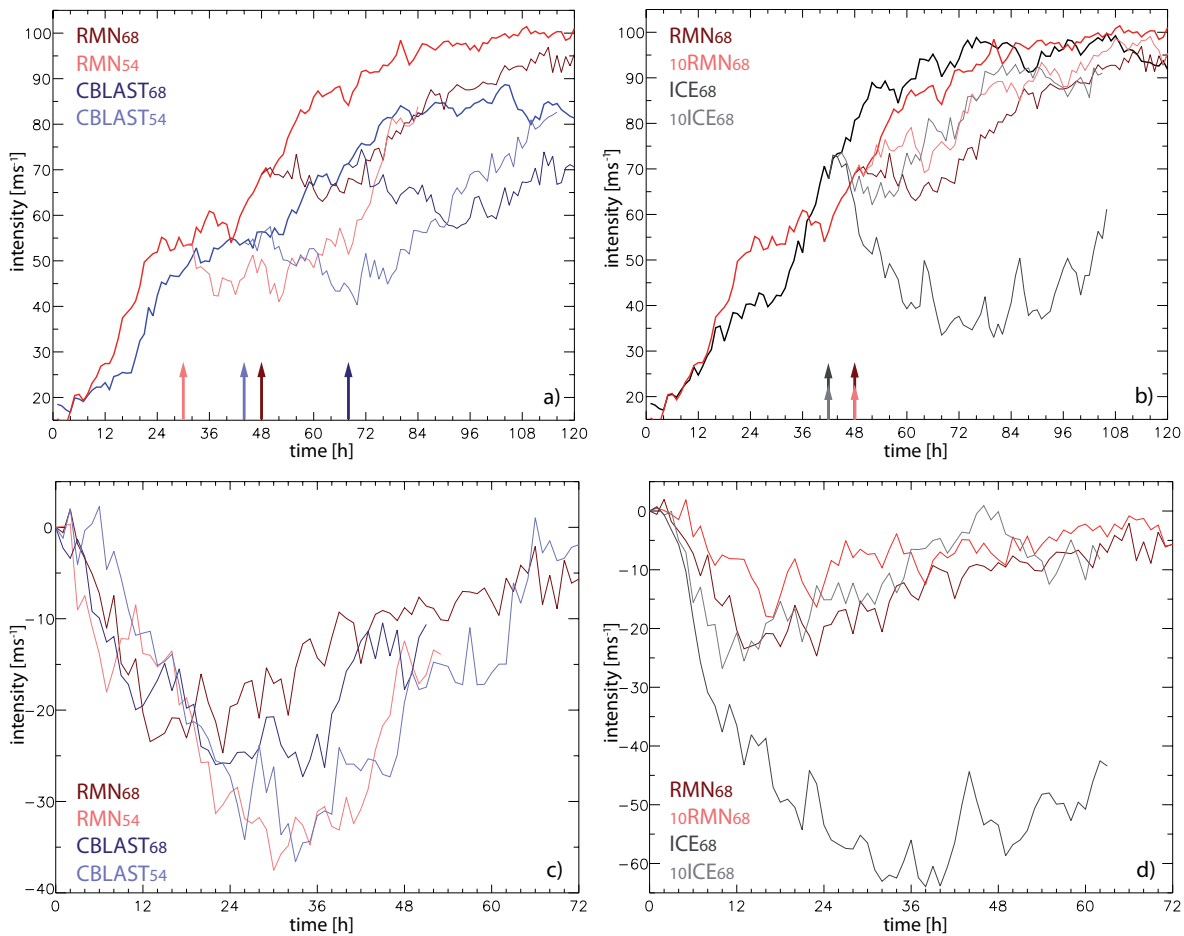

Fig. 2. (a) Intensity time series for the RMN no-shear experiment (red), $\mathrm{RMN}_{54}$ (light red), $\mathrm{RMN}_{68}$ (dark red), the CBLAST no-shear experiment (blue), CBLAST $_{54}$ (light blue), and CBLAST $_{68}$ (dark blue). The colour-coded arrows denote the time when vertical wind shear is imposed in the respective experiment. (b) As in (a), but for the RMN no-shear experiment (red), $\mathrm{RMN}_{68}$ (dark red), ${ }_{10} \mathrm{RMN}_{68}$ (light red), the ICE no-shear experiment (black), $\mathrm{ICE}_{68}$ (dark grey), and ${ }_{10} \mathrm{ICE}_{68}$ (light grey). (c) Time series of the difference between the intensity in $\mathrm{RMN}_{54}$ (light red), $\mathrm{RMN}_{68}$ (dark red), $\mathrm{CBLAST}_{54}$ (light blue), and $\mathrm{CBLAST}_{68}$ (dark blue) and the respective no-shear experiment. Time $0 \mathrm{~h}$ denotes the time when vertical wind shear is imposed in the respective experiment. (d) As in (c), but for $\mathrm{RMN}_{68}\left(\mathrm{dark}\right.$ red), ${ }_{10} \mathrm{RMN} 68$ (red), $\mathrm{ICE}_{68}$ (dark grey), and ${ }_{10} \mathrm{ICE}_{68}$ (light grey).

comparison of the vortex structure at the time when vertical wind shear is imposed. The radial, azimuthal-mean profiles of the swirling winds at this time are illustrated in Fig. 1. In CBLAST $_{68}$, the radius of maximum winds (RMW) is found at $40 \mathrm{~km}$ (Fig. 1a). The vorticity maximum is $35 \times 10^{-4} \mathrm{~s}^{-1}$ at a radius of $20 \mathrm{~km}$. This vortex structure is very similar as in RMN $_{68}$ (shown in Fig. 4 in RMN10) ${ }^{11}$. For the weaker TC in CBLAST $_{54}$, the vorticity structure outside a radius of $35 \mathrm{~km}$ is almost identical to CBLAST $_{68}$. Inside this radius, vorticity is virtually constant with a value of approx. $20 \times 10^{-4} \mathrm{~s}^{-1}$. The corresponding RMW is found at $48 \mathrm{~km}$. The initial radial structure of the TC in $\mathrm{RMN}_{54}$ is very similar (not shown).

The vorticity structure of the TC in $\mathrm{ICE}_{68}$ exhibits some notable differences as compared to the warm-rain experiments CBLAST $_{68}$ and $\mathrm{RMN}_{68}$. The vorticity maximum is found inside a radius of $10 \mathrm{~km}$ with values of $55 \times 10^{-4} \mathrm{~s}^{-1}$, i.e. approx. $60 \%$ higher than in CBLAST $_{68}$. Radially outside of this maximum, the high vorticity values decrease rapidly.

\footnotetext{
${ }^{11}$ A potential impact of barotropic instability associated with the reversal of the radial vorticity gradient in the inner core on the intensity evolution of the sheared TCs in our experiments is arguably negligible (see Sect. 3.1 in RMN10).
}

Corresponding to the smaller radius of maximum vorticity, the RMW is smaller (located at $30 \mathrm{~km}$ ) than in $\mathrm{CBLAST}_{68}$ and $\mathrm{RMN}_{68}$ also.

As discussed above, the resiliency of the TC vortex may crucially depend on the gradient of (potential) vorticity at the critical radius of the (near-discrete) VRW mode that represents the vortex tilt. The critical radius is found where the azimuthal propagation of this VRW, i.e. the vortex precession, equals the speed of the swirling winds. The precession rate, and thus the critical radius, in our experiments is difficult to determine. After the vortices have re-aligned, a short precession period of $2-3 \mathrm{~h}$ is indicated in all experiments (see Fig. 4 below $^{12}$ ). Using the values of the axisymmetric swirling winds at $1 \mathrm{~km}$, as depicted in Fig. 1a, our rough estimate of the critical radius is then $50-90 \mathrm{~km}$.

In ICE, the vorticity gradient is near-zero between $50 \mathrm{~km}$ and $65 \mathrm{~km}$ while in $\mathrm{CBLAST}_{68}$ and $\mathrm{RMN}_{54}$ the gradient is negative and of large magnitude (Fig. 1b). Between $65 \mathrm{~km}$ and $100 \mathrm{~km}$ the radial vorticity profiles are broadly similar. At larger radii (not shown) the average vorticity gradient is

\footnotetext{
${ }^{12}$ Note that the temporal resolution of the data in Fig. 4 is $1 \mathrm{~h}$. It is thus most likely that the vortex precession is undersampled.
} 
positive in $\mathrm{RMN}_{54}$ (between $120 \mathrm{~km}$ and $200 \mathrm{~km}$ ) and ICE (between 150-200 km). In contrast, in CBLAST, vorticity decreases monotonically out to $200 \mathrm{~km}$ radius. It is questionable, however, whether these differences at a radius considerably larger than our estimate for the critical radius play a role for the TC-vortex evolution in our experiments.

From the discussion in this subsection it is clear that differences in the evolution of the TCs when interacting with the vertical shear flow in the various experiments potentially arise due to differences in the radial vorticity profiles that exist before shear is imposed. The weaker vortices in $\mathrm{RMN}_{54}$ and $\mathrm{CBLAST}_{54}$, as well as the smaller-scale vortex in $\mathrm{ICE}_{68}$, can be expected to be more susceptible to tilting than their stronger, respectively broader-scale counterparts in $\mathrm{RMN}_{68}$ and CBLAST $_{68}$. In $\mathrm{ICE}_{68}$, the susceptibility to tilting is potentially enhanced by the near-zero gradient of the skirt vorticity inside of $65 \mathrm{~km}$. In Sect. 3.3 it will be shown that the tilt evolution is broadly consistent with these expectations. Furthermore, based on the results of Wong and Chan (2004), summarized at the end of Sect. 3.1.1, a more pronounced intensity decrease in $\mathrm{ICE}_{68}$ can be expected due to the smaller radial scale of the vortex alone. The latter point needs to be borne in mind when attempting to attribute differences between $\mathrm{ICE}_{68}$ and $\mathrm{RMN}_{68}$ to their different representation of cloud microphysical processes.

\subsection{Intensity evolution}

An overview of the intensity evolution in our experiments is presented in Fig. 2. First, a brief account of the intensity evolution in the quiescent environment, i.e. without vertical shear, is given. The intensity evolution in RMN is described in some detail in RMN10. In CBLAST (Fig. 2a), the incipient TC slowly intensifies to $25 \mathrm{~m} \mathrm{~s}^{-1}$ in the first $20 \mathrm{~h}$. A period of rapid intensification ensues during which the TC reaches an intensity of $53 \mathrm{~m} \mathrm{~s}^{-1}$ at $33 \mathrm{~h}$. In the subsequent $20 \mathrm{~h}$, the TC consolidates and slightly intensifies to $56 \mathrm{~m} \mathrm{~s}^{-1}$. Then, the TC continues to intensify at a moderate rate until it reaches a quasi-steady intensity of $80-85 \mathrm{~m} \mathrm{~s}^{-1}$ at $80 \mathrm{~h}$. In ICE (Fig. 2b), the TC intensifies to approx. $40 \mathrm{~m} \mathrm{~s}^{-1}$ at $30 \mathrm{~h}$ after a short gestation period. Very rapid intensification ensues and the intensity reaches $90 \mathrm{~m} \mathrm{~s}^{-1}$ at $50 \mathrm{~h}$. The maximum, quasi-steady intensity of $95-100 \mathrm{~m} \mathrm{~s}^{-1}$ is reached around $72 \mathrm{~h}$.

In all experiments, intensity decreases after vertical wind shear is imposed. As in RMN10, all TCs are resilient to the detrimental impact of vertical wind shear. After a period of weakening the TCs re-intensify; particularly rapidly in $\mathrm{RMN}_{54}{ }^{13}$. The re-intensification process in this experimental configuration is discussed in some detail in RMN10 (their Sect. 6).

\footnotetext{
${ }^{13}$ Because the focus of this study is on the structural changes of the TCs during the weakening phase, this experiment was discontinued at $84 \mathrm{~h}$.
}

To facilitate the intercomparison of the intensity evolution in the various experiments, the intensity difference from the respective no-shear experiment is presented in Fig. 2c, d. Time $0 \mathrm{~h}$ in these figures denotes the time when vertical wind shear is imposed in the respective experiment. In all warmrain experiments (Fig. 2c), the characteristics of this relative weakening are similar in the first $20 \mathrm{~h}$. The only exception being that the weakening in CBLAST $_{54}$ is delayed by $4 \mathrm{~h}$. This detail will be examined in Sect. 3.6.2. After $20 \mathrm{~h}$, the experiments with weaker TCs $\left(\mathrm{RMN}_{54}\right.$ and $\left.\mathrm{CBLAST}_{54}\right)$ continue to weaken. In contrast, CBLAST $_{68}$ maintains its intensity difference for the next $20 \mathrm{~h}$ and $\mathrm{RMN}_{68}$ re-intensifies. Based on these plots alone it might therefore be concluded that the weaker storms are more susceptible to shear. However, this is not necessarily the case here. Note that $20 \mathrm{~h}$ after shear is imposed in $\mathrm{RMN}_{54}$ and $\mathrm{CBLAST}_{54}$ the respective reference TCs in a quiescent environment are still rapidly intensifying. In contrast, the reference TCs in $\mathrm{RMN}_{68}$ and CBLAST 68 intensify only slowly $20 \mathrm{~h}$ after vertical wind shear is imposed. Thus, the differences in the relative intensity evolution partly arise due to the differences in the contemporaneous evolution of the reference TC. This brief discussion illustrates one of the inevitable challenges to interpreting different TC simulations in vertical wind shear.

The comparison of the RMN and ICE shear experiments is less complicated because the intensity evolution of the reference runs in quiescent environment is similar (Fig. 2b, d). A comparison of the intensity evolution in ${ }_{10} \mathrm{ICE}_{68}$ and $\mathrm{ICE}_{68}$, respectively, clearly shows that the TCs in the ICE experiments are more susceptible to shear than their counterparts in the warm-rain experiments. The differences between the relative weakening in ${ }_{10} \mathrm{ICE}_{68}$ and ${ }_{10} \mathrm{RMN}_{68}$, i.e. the experiments with imposed vertical shear of $10 \mathrm{~m} \mathrm{~s}^{-1}$, is on average approx. $10 \mathrm{~m} \mathrm{~s}^{-1}$ before $16 \mathrm{~h}$, and on average approx. $5 \mathrm{~m} \mathrm{~s}^{-1}$ between $16 \mathrm{~h}$ and $36 \mathrm{~h}$ (Fig. 2d). After this time, it is no longer evident that the weakening in ${ }_{10} \mathrm{ICE}_{68}$ is more pronounced than in ${ }_{10} \mathrm{RMN}_{68}$.

For $15 \mathrm{~m} \mathrm{~s}^{-1}$ shear, the difference in the intensity evolution between the ice and warm-rain experiments is striking. The maximum relative weakening in $\mathrm{ICE}_{68}$ is $-60 \mathrm{~m} \mathrm{~s}^{-1}$ (Fig. 2d). The TC reaches a minimum intensity of $35 \mathrm{~m} \mathrm{~s}^{-1}$ (Fig. 2b). For $\mathrm{RMN}_{68}$, the respective values are $-25 \mathrm{~m} \mathrm{~s}^{-1}$ and $63 \mathrm{~m} \mathrm{~s}^{-1}$. Notwithstanding this distinct intensity evolution, it will be shown below that the structural changes in $\mathrm{ICE}_{68}$ and $\mathrm{RMN}_{68}$ are very similar. In particular, the pronounced weakening in $\mathrm{ICE}_{68}$ is associated with by far the most significant depression of inflow layer $\theta_{\mathrm{e}}$ (Sects. 3.6 and 4.1). The evolution in $\mathrm{ICE}_{68}$ is thus consistent with the results of RMN10, i.e. the shear-induced frustration of inflow layer $\theta_{\mathrm{e}}$ plays a crucial role in the intensity evolution in $\mathrm{ICE}_{68}$. Furthermore, it will be argued in Sect. 4.2 that this rapid weakening is consistent with the frictional spindown of an axisymmetric vortex due to divergence above the inflow layer associated with a significant reduction of the inner-core convective mass flux. 

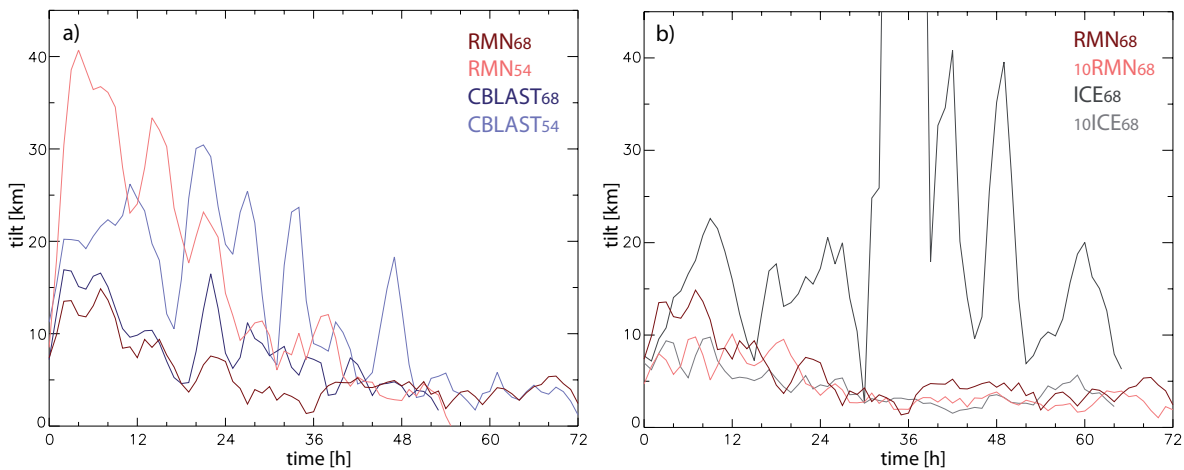

Fig. 3. (a) Time series of the tilt magnitude for $\mathrm{RMN}_{54}$ (light red), $\mathrm{RMN}_{68}$ (dark red), CBLAST 54 (light blue), and $\mathrm{CBLAST}_{68}$ (dark blue). Time $0 \mathrm{~h}$ denotes the time when vertical wind shear is imposed in the respective experiment. Vortex tilt is defined as the vector difference between the vorticity centroids at $10 \mathrm{~km}$ and $1 \mathrm{~km}$ height. For visual clarity, the $3 \mathrm{~h}$ running mean of the time series is shown. (b) As in (a) but for $\mathrm{RMN}_{68}$ (dark red), ${ }_{10} \mathrm{RMN}_{68}$ (light red), $\mathrm{ICE}_{68}$ (dark grey), and ${ }_{10} \mathrm{ICE}_{68}$ (light grey). Note that the tilt magnitude for ICE 68 goes off the scale between $33 \mathrm{~h}$ and $38 \mathrm{~h}$ reaching a maximum value of $195 \mathrm{~km}$ (not shown).
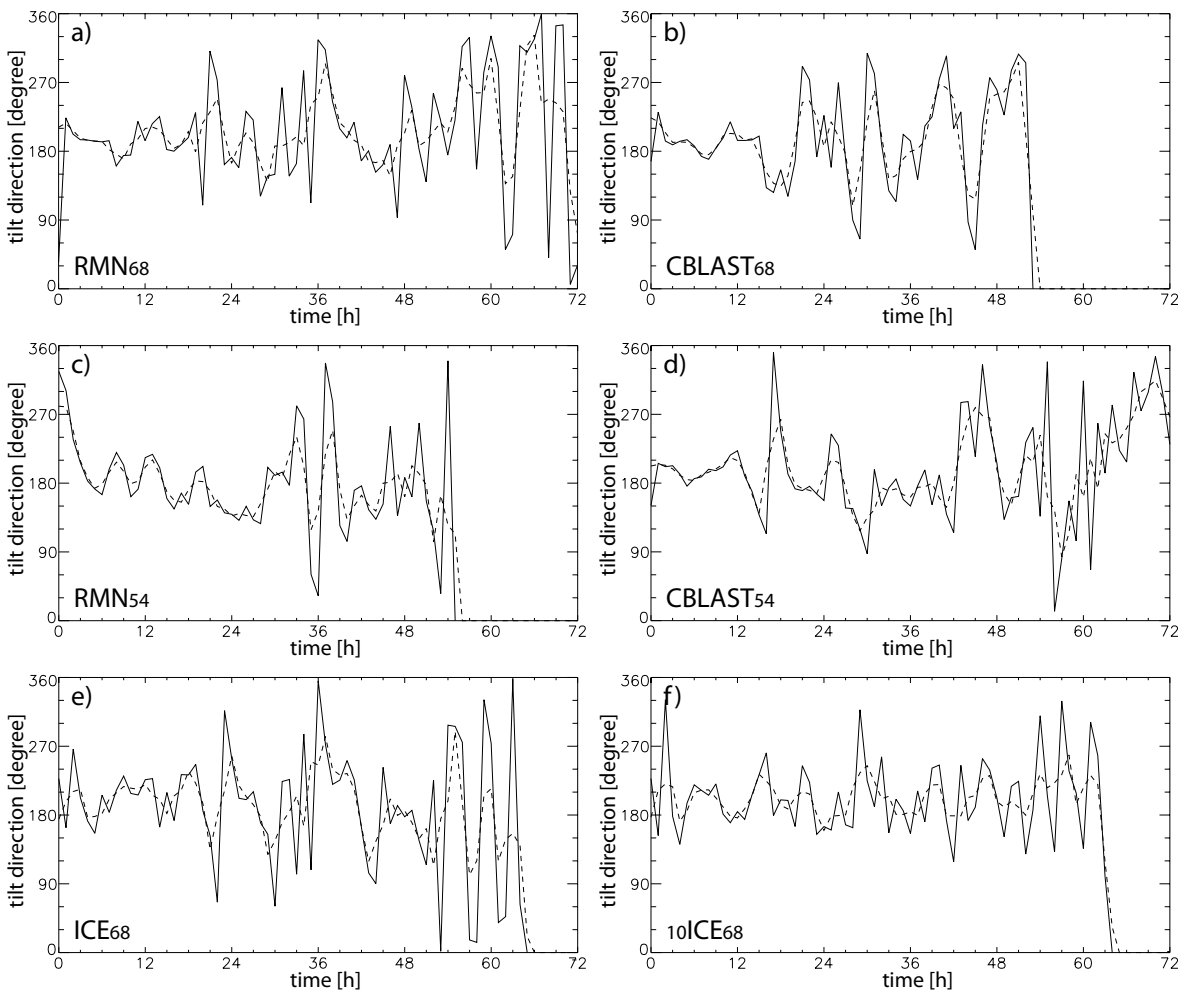

Fig. 4. Time series of the tilt direction for $\operatorname{RMN}_{68}$ (a), $\operatorname{CBLAST}_{68}(\mathbf{b}), \mathrm{RMN}_{54}$ (c), $\mathrm{CBLAST}_{54}(\mathbf{d}), \mathrm{ICE}_{68}(\mathbf{e})$, and ${ }_{10} \mathrm{ICE}_{68}(\mathbf{f})$. Time $0 \mathrm{~h}$ denotes the time when vertical wind shear is imposed in the respective experiment. Vortex tilt is defined as the vector difference between the vorticity centroids at $10 \mathrm{~km}$ and $1 \mathrm{~km}$ height. Dashed line denotes the $3 \mathrm{~h}$ running mean. Note that the temporal resolution $(1 \mathrm{~h})$ of the data is too coarse to resolve a putative high-frequency precession of the TC vortex.

\subsection{Evolution of vortex tilt}

Following RMN10, the tilt of the vortex is defined here as the vector difference between the vorticity centroids at $10 \mathrm{~km}$ and $1 \mathrm{~km}$ height. Details on the calculation of the vorticity centroids are given in RMN10. Here, the tilt magnitude and the tilt direction in our experiments are depicted in Figs. 3 and 4 , respectively.

A detailed examination of the tilt evolution in $\mathrm{RMN}_{68}$ was given in Sect. 5.1 of RMN10. There, evidence was presented that the tilt evolution is governed to zero order by asymmetric-balance dynamics. The $\mathrm{TC}$ in $\mathrm{RMN}_{68}$ settles 
into a quasi-equilibrium tilt direction to the downshear-left (south) shortly after shear is imposed (approx. within $3 \mathrm{~h}$ ). A quasi-equilibrium tilt direction may be achieved when the cyclonic precession of the tilted vortex cancels the differential advection by the vertical shear flow. This quasiequilibrium vortex tilt can be described in a first approximation by the balanced dynamics of an initially barotropic, dry TC-like vortex (Jones, 1995; Reasor et al., 2004). In $\mathrm{RMN}_{68}$, the tilt amplitude decreases while maintaining the quasi-equilibrium tilt direction. The TC virtually re-aligns within approx. $24 \mathrm{~h}$, i.e. after this time the tilt amplitude is comparable to the tilt amplitude of the TC in the no-shear benchmark experiment (see Fig. 5 in RMN10). Once the tilt amplitude has decreased considerably, a high variability in the tilt direction ensues, indicative of a high-frequency precession of the vortex (Fig. 4a).

Figures 3 and 4 demonstrate that the general characteristics of the tilt evolution are similar in all of our experiments in the first $24-30 \mathrm{~h}$ after vertical wind shear is imposed. All TCs are resilient during this time and the tilt direction is predominantly to the South $\left(180^{\circ}\right)$. In CBLAST $_{68}$, the maximum tilt magnitude, as well as the temporal tilt evolution, is very similar to that of $\mathrm{RMN}_{68}$. The only exception being that the local maximum at $23 \mathrm{~h}$ is more pronounced and, thereafter, the tilt magnitude remains $3-5 \mathrm{~km}$ larger. As can be expected from the discussion in Sect. 3.1, the weaker TCs in $\mathrm{RMN}_{54}$ and CBLAST $_{54}$ clearly exhibit a larger tilt magnitude in the first $24 \mathrm{~h}$ than the more intense TCs in $\mathrm{RMN}_{68}, \mathrm{CBLAST}_{68}$, and $\mathrm{ICE}_{68}$. Maximum tilt amplitudes reach $40 \mathrm{~km}$ in $\mathrm{RMN}_{54}$ and $30 \mathrm{~km}$ in CBLAST $_{54}$. Both TCs eventually re-align.

The most striking feature in the tilt evolution is the disruption of the TC vortex after $30 \mathrm{~h}$ in $\mathrm{ICE}_{68}$ (Fig. 3). Until this time the TC tends to re-align slowly: the local tilt maximum and minimum at $25 \mathrm{~h}$ and $30 \mathrm{~h}$, respectively, are smaller than their respective predecessors at $9 \mathrm{~h}$ and $15 \mathrm{~h}$. At $30 \mathrm{~h}$, intensity has decreased by over $30 \mathrm{~m} \mathrm{~s}^{-1}$ as compared to the time when vertical wind shear is imposed and has reached the smallest absolute value in all experiments (approx. $35 \mathrm{~m} \mathrm{~s}^{-1}$, Fig. 2). Based on the discussion in Sect. 3.1, it is plausible that the significantly weakened TC can no longer withstand the differential advection associated with the vertical wind shear. Note that the distinct weakening in the first $20 \mathrm{~h}$ precedes the disruption of the TC by more than $10 \mathrm{~h}$. After $30 \mathrm{~h}$, the TC remains as a coherent entity with smaller vertical extent: the tilt as inferred from the vorticity centroid at $9 \mathrm{~km}$ height instead of $10 \mathrm{~km}$ height does not exceed $35 \mathrm{~km}$ (not shown). Arguably, the survival as a vertically less extensive system allows for the rapid re-intensification of the TC after the inflow layer $\theta_{\mathrm{e}}$ values have recovered (Sect. 4.1).

The tilt magnitude in ${ }_{10} \mathrm{ICE}_{68}$ remains below $10 \mathrm{~km}$ and quickly reduces to values smaller than $6 \mathrm{~km}$ after $12 \mathrm{~h}$. Despite this small tilt magnitude a southerly tilt direction is preferred until late in the experiment. Although the evolution of the tilt magnitude is similar in ${ }_{10} \mathrm{RMN}_{68}$, the tilt direction in ${ }_{10} \mathrm{RMN}_{68}$ exhibits more variability after $30 \mathrm{~h}$ when the tilt magnitude is small (not shown).

\subsection{SBC formation and downward flux of low- $\theta_{\mathrm{e}}$ air into the inflow layer}

A metric for the downward flux of low- $\theta_{\mathrm{e}}$ air into the inflow layer (DFX) has been defined in RMN10 (their Sect. 4.2.1) as ${ }^{14}$

$\mathrm{DFX} \equiv w_{-} \theta_{\mathrm{e}}^{\prime}$

where $w_{-}$denotes downward vertical motion and $\theta_{\mathrm{e}}^{\prime}$ the perturbation from the azimuthal mean of $\theta_{\mathrm{e}}$. The quantity DFX is evaluated at $1.5 \mathrm{~km}$, the approximate top of the axisymmetric inflow layer.

The time-averaged structure of the SBC, represented by low-level upward motion, and the distribution of DFX shortly after the time when vertical wind shear is imposed is depicted in Fig. 5. In this early stage of the evolution, the general structure of the SBC and the distribution of DFX is consistent in all experiments. The SBC forms in the downshearright quadrant ${ }^{15}$ extending radially outwards to $150-200 \mathrm{~km}$. Small to moderate DFX values occur over a broad, banded region downwind of the SBC, from the downshear quadrant through downshear-left, and into the upshear quadrant. In all experiments, azimuthal vertical cross-sections (as Fig. 18 in RMN10, not shown here) indicate that the associated downdrafts form underneath the helical updrafts of the SBC.

It is of interest to note that the SBCs in $\mathrm{RMN}_{54}, \mathrm{ICE}_{68}$, and ${ }_{10} \mathrm{ICE}_{68}$, respectively, extend azimuthally further into the upshear quadrant than their counterparts in $\mathrm{RMN}_{68}$, CBLAST $_{68}$, and CBLAST $_{54}$. In the former experiments, the tilt direction at early times exhibits a greater downshear (westward) component than in the latter experiments (Fig. 4). Thus, the low-level vorticity anomaly associated with the tilted vortex can be expected to extend into the upshear quadrant. RMN10 have shown evidence that frictional convergence due to the low-level vorticity anomaly associated with the tilted vortex contributes to the forcing of the SBC. The correlation of the location of SBC formation with the tilt direction in our experiments presented here provides further support for RMN10's interpretation.

\footnotetext{
${ }^{14}$ Our downward-flux metric DFX is virtually identical to that part of the flux term $\theta_{\mathrm{e}}^{\prime} w^{\prime}$ for which $w^{\prime}$ is negative (see RMN10, their Sect. 4.2.1, for details).

${ }^{15}$ To describe the azimuthal location of relevant features in this study the following convention is used: First, the centerline of the azimuthal location is given with respect to the shear vector. Then, the azimuthal extent is given, e.g. quadrant or semicircle. For example, let $0^{\circ}$ denote north and let the shear vector point to the west $\left(270^{\circ}\right)$. Then, "downshear-right" is at $0^{\circ}$ and the "downshear-right quadrant" denotes the region between $315^{\circ}$ and $45^{\circ}$. Note that this convention differs somewhat from the one that is frequently used in the TC community.
} 

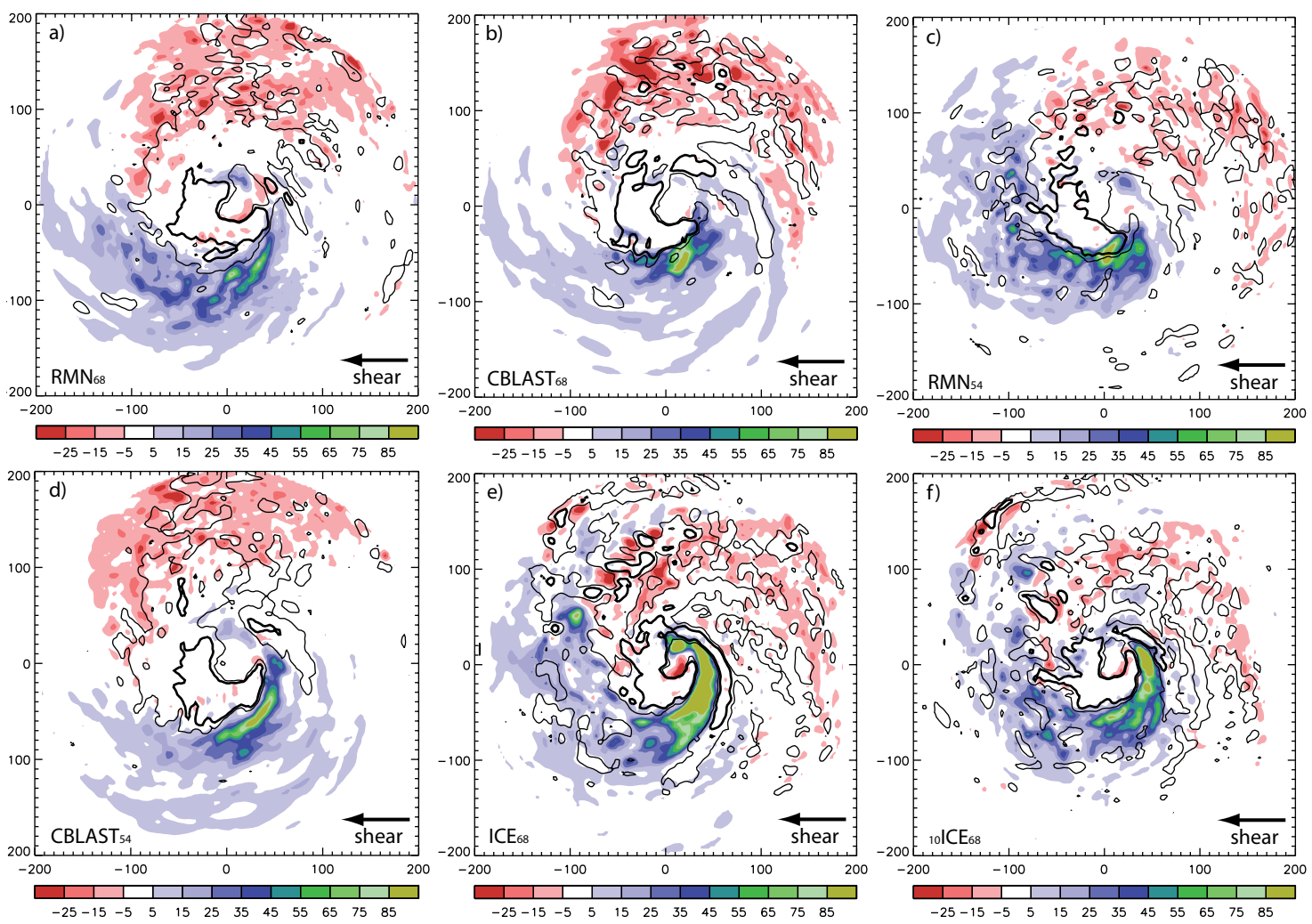

Fig. 5. Downward flux of $\theta_{\mathrm{e}}^{\prime}$, DFX (colour, in $0.1 \mathrm{~K} \mathrm{~m} \mathrm{~s}^{-1}$, see text for definition), at the top of the axisymmetric inflow layer, defined as $1.5 \mathrm{~km}$, and low-level upward motion (thin contour: $0.25 \mathrm{~m} \mathrm{~s}^{-1}$, thick contour: $1 \mathrm{~m} \mathrm{~s}^{-1}$, averaged from $1.25-2 \mathrm{~km}$ height) both averaged from 1-6 h after vertical shear is imposed for (a) $\mathrm{RMN}_{68}$, (b) $\mathrm{CBLAST}_{68}$, (c) $\mathrm{RMN}_{54}$, (d) $\mathrm{CBLAST}_{54}$, (e) $\mathrm{ICE}_{68}$, and (f) ${ }_{10} \mathrm{ICE}_{68}$. The arrow indicates the direction of the deep-layer shear. The horizontal scale is in $\mathrm{km}$.

A distinct maximum of DFX is found in the upshear quadrant close to the eyewall. This maximum is most pronounced in the ICE experiments (Fig. 5e, f). In RMN10, this DFX maximum was not given special attention. A pronounced maximum in the upshear quadrant occured in the "20mps" case of RMN10 (their Fig. 10d). RMN10 further noted a localized region of downdrafts in the upshear region close to the eyewall after the TCs in the "10mps" and "15mps" cases have re-aligned (their Fig. 11a and associated discussion).

The DFX maximum is located at the downwind end of the banded region of positive DFX values. A pronounced $\theta_{\mathrm{e}}$ depression is found at the top of the inflow layer in this region (not shown). Therefore, large negative values of $\theta_{\mathrm{e}}^{\prime}$ contribute to high values of DFX (Eq. 4). However, downward motion into the inflow layer is particularly strong in this region also (not shown). A preliminary examination of the distributions of vertical motion, hydrometeors, and $\theta_{\mathrm{e}}$ suggests that the downdrafts close to the eyewall may form by evaporation of precipitation falling out of the tilted eyewall. To us, however, it is not clear whether the more pronounced localized maximum in the ICE experiments is solely due to the inclusion of ice microphysics or to what extent the differences in the radial structure of the TC vortex contribute. Clearly, more re- search is needed to clarify the formation mechanism of these downdrafts, as well as the relative importance of the confined DFX maximum and the broad region of moderate DFX for TC intensity evolution.

\subsection{Vortex-tilt induced dynamic instability?}

A non-linear, Kelvin-Helmholtz type instability in the inner core of a tilted, dry TC-like vortex has been found in a numerical experiment by Reasor et al. (2004, their Sect. 5a). In their experiment, this instability contributed to enhanced localized, three-dimensional mixing. Such mixing is one pathway to dissipate kinetic energy in the TC's inner core. The role of this mixing for the intensity evolution of TCs in vertical wind shear is hitherto unexplored.

The necessary condition for such an instability is that the gradient Richardson number, $\mathcal{R}$, drops below unity (Abarbanel et al., 1986). Here, $\mathcal{R}$ is calculated as

$$
\mathcal{R}=N^{2} /\left((d u / d z)^{2}+(d v / d z)^{2}\right),
$$



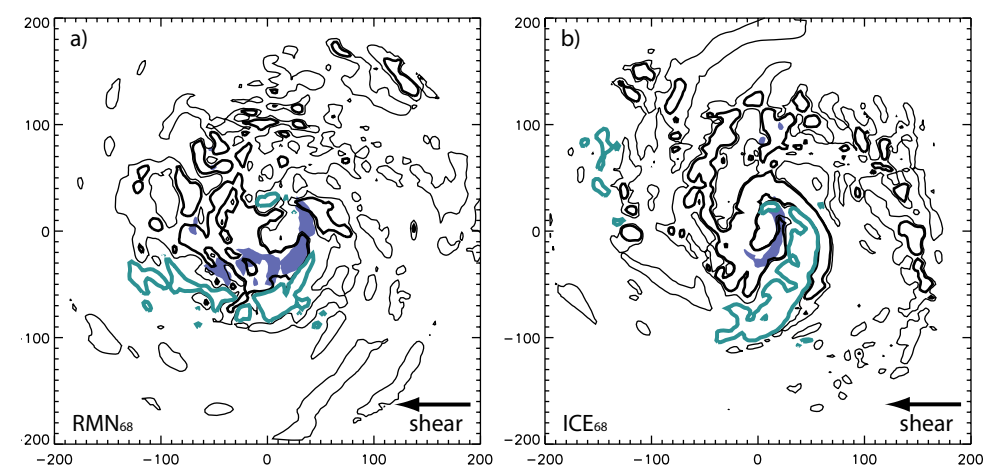

Fig. 6. Snapshots of the gradient Richardson number at $1625 \mathrm{~m}$ height and $3 \mathrm{~h}$ after vertical shear is imposed (shaded for values below 1 ) in (a) $\mathrm{RMN}_{68}$ and (b) $\mathrm{ICE}_{68}$. Upward motion is depicted in black contours (thin for $0.25 \mathrm{~m} \mathrm{~s}^{-1}$, thick for $1 \mathrm{~m} \mathrm{~s}^{-1}$ ). The cyan contour denotes regions of strong downward flux of low- $\theta_{\mathrm{e}}$ air into the inflow layer (DFX at $1.5 \mathrm{~km}$ height, the contour depicts the $4.5 \mathrm{~K} \mathrm{~m} \mathrm{~s}{ }^{-1}$ isoline). The horizontal scale is in $\mathrm{km}$.

where $N$ is the (dry) vertical static stability, and $u$ and $v$ are the radial and tangential wind components ${ }^{16}$, respectively. The tilt of the vortex may lead to a high local vertical shear of the swirling winds in the inner core. Thus, in such regions, the gradient Richardson number may reach small values.

In all of our (moist) TC experiments, the potential for a Kelvin-Helmholtz type instability and the associated threedimensional, turbulent mixing in the inner core is an element of vertical-shear interaction. Underneath the eyewall and the SBC, $\mathcal{R}<1$ is an ubiquitous feature within the layer of strong, frictionally-driven inflow (not shown). Above this inflow layer, localized regions of $\mathcal{R}<1$ occur, at least temporarily, in all experiments presented in this study within the first $6 \mathrm{~h}$ after shear is imposed. These regions are located within the eyewall in the downshear-left to upshear quadrant (exemplified in Fig. 6 for $\mathrm{RMN}_{68}$ and $\mathrm{ICE}_{68}$ ). This feature is most pronounced in $\mathrm{RMN}_{68}$ and least pronounced in $\mathrm{RMN}_{54}$, as might be expected for the weaker vortex in $\mathrm{RMN}_{54}$. The regions of $\mathcal{R}<1$ are frequently located radially inwards (within 20-30 km) of regions of high DFX (exemplified in Fig. 6). Due to this proximity, it seems justified to hypothesize that enhanced mixing associated with the tilt-induced instability locally modifies the downward flux of low- $\theta_{\mathrm{e}}$ air into the inflow layer. A quantitative diagnostic of this mixing process is deferred to future work.

\subsection{Inflow layer $\theta_{\mathrm{e}}$ depression}

\subsection{1 $\theta_{\mathrm{e}}$ depression outside of the eyewall}

A notable depression of $\theta_{\mathrm{e}}$ values in the inflow layer outside of the eyewall occurs in all experiments $2-3 \mathrm{~h}$ after shear is imposed. A snapshot of the height-averaged inflow layer $\theta_{\mathrm{e}}$ distribution representative of the early part of the shear ex-

\footnotetext{
${ }^{16}$ Normally, the definition of $\mathcal{R}$ involves the zonal and meridional wind components. Our calculation of $\mathcal{R}$ is equivalent to this definition.
}

periments (at $5 \mathrm{~h}$ ) is presented in Fig. 7. As can be expected from the DFX patterns discussed above, the inflow layer $\theta_{\mathrm{e}}$ distribution is similar in all experiments at this time: Low$\theta_{\mathrm{e}}$ air wraps from the downshear quadrant into the upshear quadrant cyclonically inward ${ }^{17}$. Also evident in all cases is the pronounced wave-number 1 signature of the SBC in the vertical motion field at low levels above the inflow layer.

To facilitate the intercomparison of the experiments we consider now the deviation of the horizontal distribution of $\theta_{\mathrm{e}}$ relative to its initial azimuthal average at the time when shear is imposed in the respective experiment (Fig. 8). This figure depicts time-averaged values from $4-9 \mathrm{~h}$ after shear is imposed. In all cases, the inflow layer $\theta_{\mathrm{e}}$ values outside of the inner-core updrafts are significantly depressed. A depression of up to $15 \mathrm{~K}$ can be found in $\mathrm{RMN}_{68}, \mathrm{RMN}_{54}$, and $\mathrm{ICE}_{68}$. Minimum $\theta_{\mathrm{e}}$ values are generally found to the southeast of the centre (upshear to downshear-left). This location is consistent with the DFX maximum in this region and the continuous depression of $\theta_{\mathrm{e}}$ values along the spiralling inflow leg, as hypothesised by RMN10 (their Fig. 11a). The inflow layer $\theta_{\mathrm{e}}$ depression extends into the downshear-right quadrant also, outwards to a radius of $100-150 \mathrm{~km}$. In the absence of downward flux of low $\theta_{\mathrm{e}}$ in this region (cf. Fig. 5) the inflow layer $\theta_{\mathrm{e}}$ values gradually recover while air parcels circle from the upshear quadrant to the upshear-right quadrant.

The inflow layer $\theta_{\mathrm{e}}$ depression outside of the eyewall in the CBLAST experiments is less pronounced than in the comparable RMN experiments. While the minima in RMN exceed $-13 \mathrm{~K}$, the minima in CBLAST have values of approx. $-10 \mathrm{~K}$. This less pronounced $\theta_{\mathrm{e}}$ depression is consistent with smaller average DFX values in CBLAST $_{68}$ and

\footnotetext{
${ }^{17}$ Approximately, this $\theta_{\mathrm{e}}$ depression is associated with a depression in the water vapor mixing ratio by $4-5 \mathrm{~g} \mathrm{~kg}^{-1}$ and a depression in the potential temperature by $2-3 \mathrm{~K}$. The spatial distribution of these fields for $\mathrm{ICE}_{68}$ and $\mathrm{RMN}_{68}$ at the time shown in Fig. 7 can be found on the ACPD webpage in the authors' response to the anonymous reviewer (item 3 ).
} 

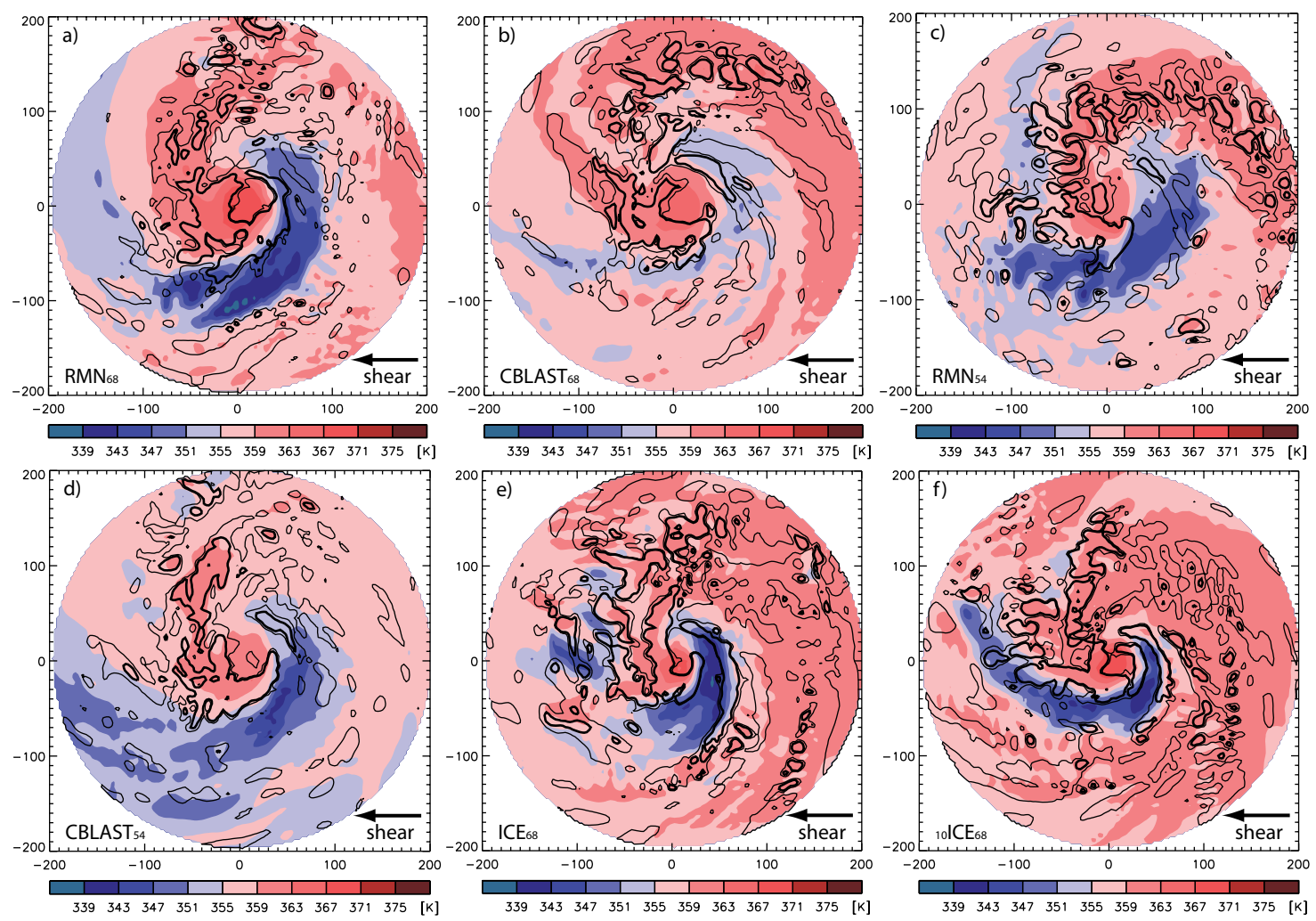

Fig. 7. As Fig. 5, but for a snapshot of inflow layer $\theta_{\mathrm{e}}$ (colour, averaged over lowest $1 \mathrm{~km}$ ) and low-level updrafts at $5 \mathrm{~h}$ after vertical shear is imposed for (a) $\mathrm{RMN}_{68}$, (b) $\mathrm{CBLAST}_{68}$, (c) $\mathrm{RMN}_{54}$, (d) $\mathrm{CBLAST}_{54}$, (e) $\mathrm{ICE}_{68}$, and (f) ${ }_{10} \mathrm{ICE}_{68}$. The depicted time is representative for the early part of the experiments.

CBLAST $_{54}$ as compared to $\mathrm{RMN}_{68}$ and $\mathrm{RMN}_{54}$, respectively (Fig. 5). The smaller DFX values in CBLAST are associated with a weaker general downdraft activity (see Sect. 3.7 and Fig. 10 for supporting evidence).

In the ICE experiments, the spatial pattern of the $\theta_{\mathrm{e}}$ depression is radially more confined than in the warm-rain experiments. This is consistent with the DFX pattern. As discussed at the end of Sect. 3.4, it is not evident to us to what extent this greater radial confinement can be attributed to differences in the cloud microphysics or to differences in the radial structure of the $\mathrm{TC}$ vortices.

\subsection{2 $\theta_{\mathrm{e}}$ depression underneath the inner-core updrafts}

An axisymmetric view of the inflow layer and eyewall $\theta_{\mathrm{e}}$ distribution is presented in RMN10. In this axisymmetric view a tongue of low $-\theta_{\mathrm{e}}$ air effectively intrudes into the inflow layer from above and radially inwards towards the eyewall. The $\theta_{\mathrm{e}}$ values of air rising in the eyewall of the sheared TCs are several degrees smaller than in the experiment without vertical shear (Fig. 9 in RMN10). The same general characteristics of the axisymmetric inflow layer and adjacent eyewall structure are found in the additional experiments considered in this study (not shown). When viewed from the sim- plified perspective of an idealised Carnot-cycle, a weakening of the sheared TCs can be expected. As noted in RMN10, however, it is an open question whether an axisymmetric viewpoint is sufficient in the presence of pronounced wavenumber 1 asymmetries in the inflow layer $\theta_{\mathrm{e}}$ distribution and the eyewall updrafts of a TC in vertical wind shear.

In the present study we complement the axisymmetric view by the examination of the horizontal distribution of inflow layer $\theta_{\mathrm{e}}$ and low-level eyewall updrafts. For the following discussion the eyewall updrafts are defined as the contiguous region within the $1 \mathrm{~m} \mathrm{~s}^{-1}$ vertical velocity isotach near the centre in Fig. 8. We consider the $\theta_{\mathrm{e}}$ values within this region to be a good approximation for the $\theta_{\mathrm{e}}$ values of air rising out of the boundary/ sub-cloud layer into the eyewall updrafts. Upon examining the results, it is obvious that in all cases, the inner-core updrafts exhibit a pronounced wavenumber 1 asymmetry and that the eyewall is not closed.

By visual inspection we estimate the average $\theta_{\mathrm{e}}$ depression underneath the eyewall (averaged from 4-9 h) to be $0 \mathrm{~K}$ for $\mathrm{CBLAST}_{54}, 2-3 \mathrm{~K}$ for $\mathrm{RMN}_{68}$ and $\mathrm{CBLAST}_{68}, 3-5 \mathrm{~K}$ for $\mathrm{RMN}_{54}$ and ${ }_{10} \mathrm{ICE}_{68}$, and 6-7 K for ICE 68 . These values correlate well with the intensity evolution of the respective TCs. The TC in $\mathrm{CBLAST}_{54}$ does not weaken until $7 \mathrm{~h}$ after shear is imposed (Fig. 2c). The TCs in $\mathrm{RMN}_{68}$ and $\mathrm{CBLAST}_{68}$ 

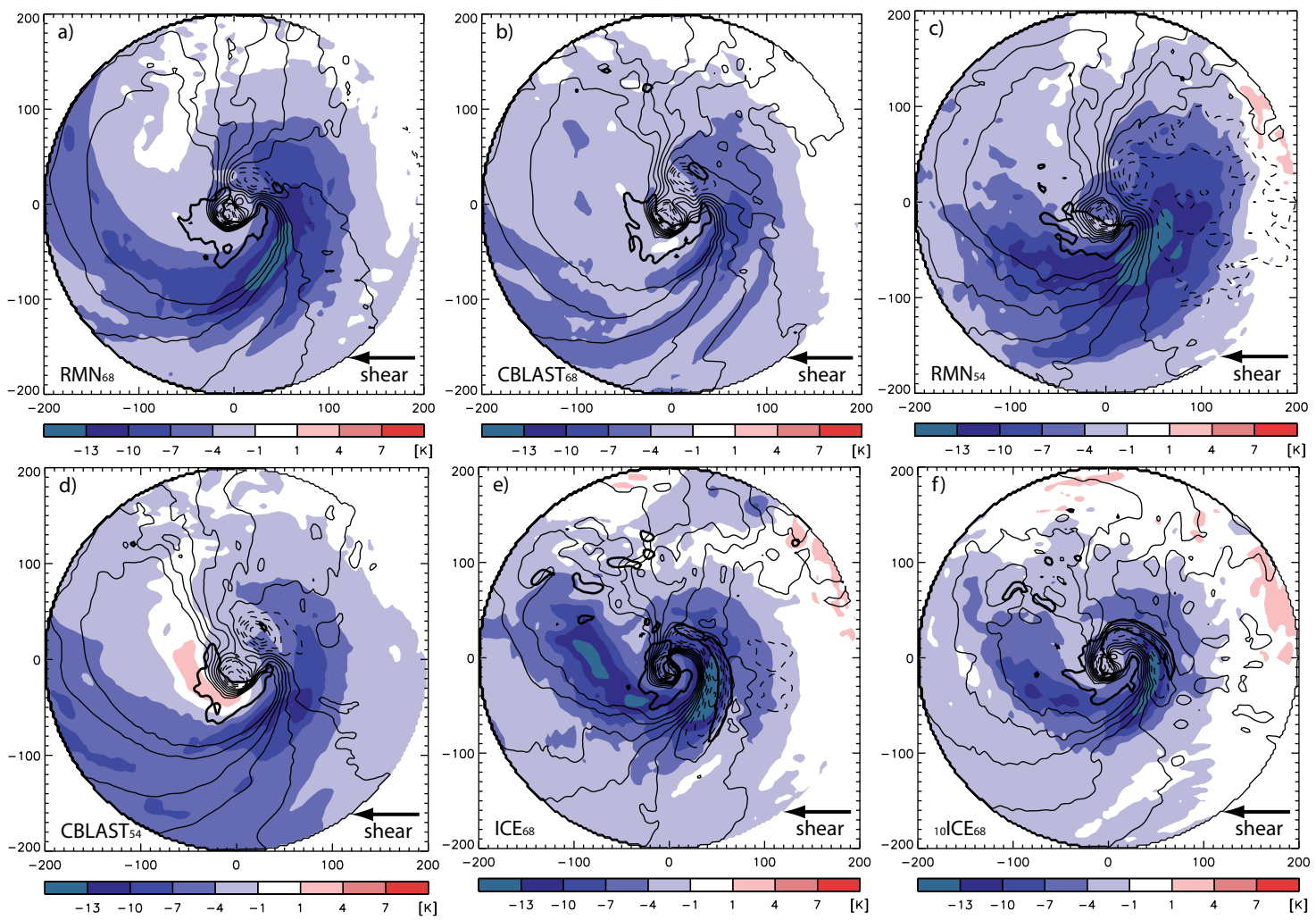

Fig. 8. Depression of inflow layer $\theta_{\mathrm{e}}$ (averaged over the lowest $1 \mathrm{~km}$ and from $4-9 \mathrm{~h}$ ) relative to the axisymmetric mean at the time when vertical wind shear is imposed (colours). Thin contours depict the radial flow averaged over the lowest $1 \mathrm{~km}\left(\mathrm{every} 4 \mathrm{~m} \mathrm{~s}^{-1}\right.$, starting at $2 \mathrm{~m} \mathrm{~s}^{-1}$, dashed for negative values (outflow)). Thick contour denotes low-level updrafts $\left(1 \mathrm{~m} \mathrm{~s}^{-1}\right.$, averaged from 1.25-2 km height). (a) $\mathrm{RMN}_{68}$, (b) $\mathrm{CBLAST}_{68}$, (c) $\mathrm{RMN}_{54}$, (d) $\mathrm{CBLAST}_{54}$, (e) $\mathrm{ICE}_{68}$, and (f) ${ }_{10} \mathrm{ICE}_{68}$.

show a very similar weakening in the first $12 \mathrm{~h}$ while $\mathrm{RMN}_{54}$ and ${ }_{10} \mathrm{ICE}_{68}$ exhibit slightly faster weakening rates. The pronounced and rapid weakening of the TC in $\mathrm{ICE}_{68}$ is associated with the most pronounced depression of $\theta_{\mathrm{e}}$ underneath the eyewall. This correlation supports RMN10's result that the intensity evolution of resilient TCs in vertical shear is governed to first order by the systematic depression of $\theta_{\mathrm{e}}$ in the inflow layer.

The inflow layer $\theta_{\mathrm{e}}$ depression outside of the eyewall in $\mathrm{CBLAST}_{68}$ is less pronounced than in $\mathrm{RMN}_{68}$. However, the $\theta_{\mathrm{e}}$ depression underneath the eyewall is very similar in both experiments. Consistently, the intensity decrease is very similar in $\mathrm{CBLAST}_{68}$ and $\mathrm{RMN}_{68}$ in the first $12 \mathrm{~h}$. Therefore, it can be inferred that the replenishment of inflow air spiralling towards the eyewall is more complete in RMN than in CBLAST. As discussed in the Introduction, the smaller value of $C_{D}$ in CBLAST may lead to weaker frictional inflow as compared to RMN and thus to a longer replenishment time. Here, this effect of a smaller $C_{D}$ value is apparently overruled by the reduced extraction of heat from the ocean surface due to a smaller value of $C_{K}$. It is difficult to examine this aspect in $\mathrm{RMN}_{54}$ and $\mathrm{CBLAST}_{54}$ because the $\theta_{\mathrm{e}}$ depres- sion underneath the eyewall is different at this early time in the experiment.

The replenishment time of air with depressed $\theta_{\mathrm{e}}$ values may depend considerably on the azimuthal location. The radial velocity averaged over the lowest $1 \mathrm{~km}$ exhibits a pronounced wave-number 1 asymmetry in all experiments (Fig. 8). In the downshear semicircle, the maximum heightaveraged radial inflow is approx. $20 \mathrm{~m} \mathrm{~s}^{-1}$ while in the upshear semicircle the height-averaged radial velocity is very small or is even directed outwards (e.g. in RMN54 (Fig. 8c)). Figure 8 indicates that the bulk of the depressed inflow layer $\theta_{\mathrm{e}}$ spirals around the centre before entering the inner core updrafts in the downshear-right quadrant (e.g. Fig. 8a, f). In contrast, in $\mathrm{RMN}_{54}$ and $\mathrm{ICE}_{68}$, an additional, much more direct pathway for low- $\theta_{\mathrm{e}}$ air from the downshear quadrant to enter the eyewall updrafts in the downshear to downshear-left quadrant is indicated (Fig. 8c, e). The replenishment of inflow layer $\theta_{\mathrm{e}}$ along this more direct pathway can be expected to be less complete than along the longer inflow spiral. In the presence of a pronounced wave-number 1 asymmetry of the inflow, it stands to reason that the (azimuthal) location of the flushing of the inflow layer with low- $\theta_{\mathrm{e}}$ air may be of 

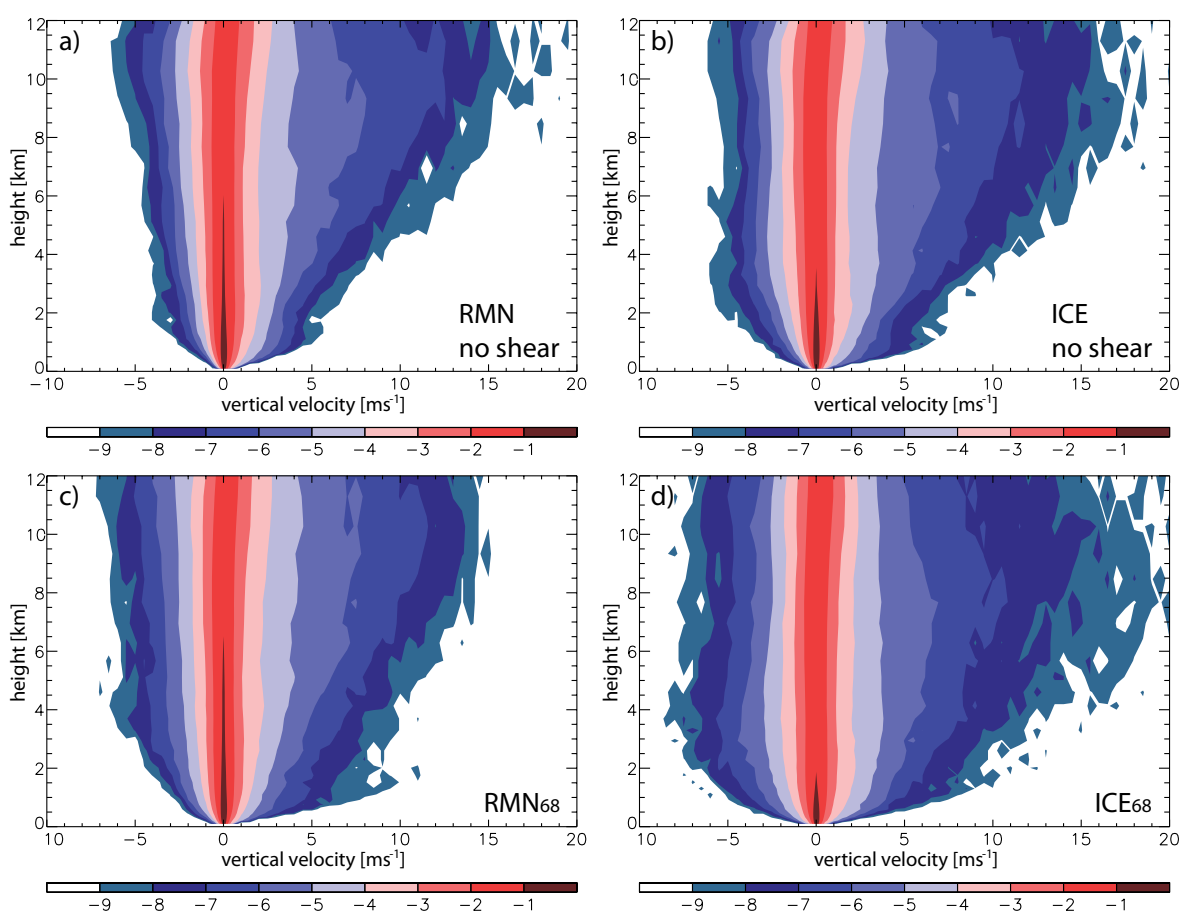

Fig. 9. Contoured frequency by altitude diagrams of vertical motion within $160 \mathrm{~km}$ radius of the centre. Vertical motion is binned into intervals of $0.5 \mathrm{~m} \mathrm{~s}^{-1}$, centred on the values given on the $x$-axis. The colour-coded value denotes the logarithm of the frequency. With each contour the frequency decreases by a factor of $1 / e$. Before taking the logarithm of the frequency a small positive value $\left(10^{-5} \approx e^{-11.5}\right)$ has been added to ensure that the logarithm is well defined everywhere. For the no-shear cases of RMN (a) and ICE (b) the $6 \mathrm{~h}$ time period after vertical wind shear is imposed in the respective shear runs is considered. For $\mathrm{RMN}_{68}$ (c) and $\mathrm{ICE}_{68}$ (d) the times 4-9 h are considered.

importance for the intensity evolution also, and not only the magnitude of the $\theta_{\mathrm{e}}$ depression in the azimuthal mean.

\subsection{Vertical motion characteristics}

The formation of the SBC and the associated downdrafts that flush low- $\theta_{\mathrm{e}}$ air into the inflow layer are two central aspects of RMN10's framework. Here, we examine the general characteristics of vertical motion in our suite of experiments using so-called contoured frequency by altitude diagrams (CFADs, Yuter and Houze, 1995). These diagrams depict the frequency of the occurrence of vertical motion of a given magnitude, here binned in $0.5 \mathrm{~m} \mathrm{~s}^{-1}$ intervals, within a given region and at a given altitude. It is very difficult to focus specifically on the SBC and on regions where shearinduced downdrafts form. Therefore, we consider all vertical motion within a radius of $160 \mathrm{~km}$.

A comparison of the RMN and ICE experiments is presented in Fig. 9. Without vertical wind shear (Fig. 9a, b), strong up- and downdrafts occur more frequently in the ICE experiment. Under the influence of vertical shear (Fig. 9c, d), the frequency distribution broadens considerably below 5-6 km as compared to the respective no-shear experiment. For both the warm rain and ice microphysics configurations, vertical wind shear increases the frequency of the strongest up- and downdrafts at midlevels and below. At upper lev- els the increase of downdraft frequency is less pronounced and the frequency of strong updrafts tends to decrease, except for the strongest updrafts in $\mathrm{ICE}_{68}$. It is evident also that strong downdrafts below $5 \mathrm{~km}$ occur considerably more frequently in $\mathrm{ICE}_{68}$ than in $\mathrm{RMN}_{68}$ (cf. Fig. 9c, d). The more pronounced downdraft activity in the ICE experiments is consistent with the tenet that the cooling associated with the melting of ice particles may initiate or strengthen existing downdrafts (Srivastava, 1987).

Whithout imposed vertical shear, the results in CBLAST are very similar to those in RMN (not shown). The imposed vertical wind shear leads to a broadening of the frequency distribution in CBLAST also (not shown). The differences between the CBLAST and RMN shear experiments are less pronounced than between ICE and RMN. The differences are depicted in Fig. 10 as the ratio of the frequencies in $\mathrm{RMN}_{68}$ and $\mathrm{CBLAST}_{68}$ (Fig. 10a) and $\mathrm{RMN}_{54}$ and CBLAST $_{54}$ (Fig. 10b). Apparently, the frequency of the strongest downdrafts is more than $275 \%$ greater in RMN than in CBLAST. Strong updrafts tend to be more frequent in $\mathrm{RMN}$ also, in particular so in $\mathrm{RMN}_{54}$ as compared to CBLAST $_{54}$.

Differences in the thermodynamic properties of the ambient air in which downdrafts occur may contribute to the observed differences in downdraft activity. The strength of 

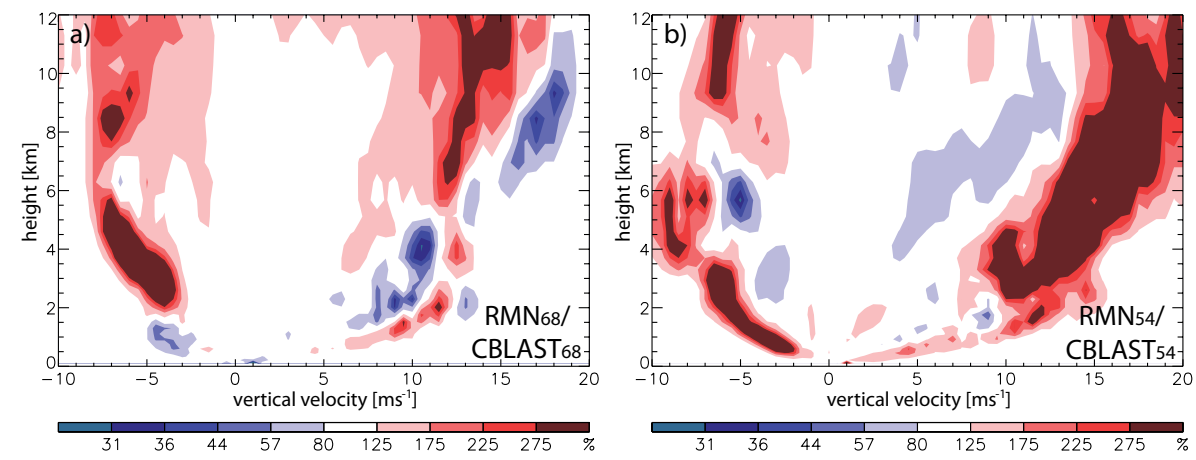

Fig. 10. As Fig. 9, but for the ratio of vertical motion frequency of $\mathrm{RMN}_{68} / \mathrm{CBLAST}_{68}$ (a) and $\mathrm{RMN}_{54} / \mathrm{CBLAST}_{54}$ (b).

a downdraft depends, inter alia, on the buoyancy difference between the descending air parcel and the ambient air along the parcel's downward path. A vertically integrated measure of this buoyancy difference is the downdraft convective available potential energy (DCAPE, Emanuel, 1994, his Sect. 6.3). The derivation of DCAPE assumes the following two-stage process. First, evaporation of precipitation cools an air parcel isobarically until saturation is reached. Then, the parcel descends pseudo-adiabatically with just enough evaporation to keep the parcel saturated.

We have calculated DCAPE using the azimuthal mean soundings at the time when shear is imposed. Because it is not entirely clear which radius is most appropriate to examine the downdrafts underneath the SBC we considered a number of different radii and radial averages between $60 \mathrm{~km}$ and $150 \mathrm{~km}$ (not shown). For none of these regions, could we find a clear relationship between the DCAPE values and the downdraft activity, or between the DCAPE values and the downward flux of low- $\theta_{\mathrm{e}}$ air into the inflow layer. In particular, DCAPE values below $500 \mathrm{hPa}$ in CBLAST $_{54}$ are higher than in $\mathrm{RMN}_{54}$, in contrast to the more pronounced downdraft activity in $\mathrm{RMN}_{54}$.

Apparently, consideration of solely thermodynamic properties of the air in which the downdrafts occur is insufficient to explain the observed differences in downdraft activity. We propose the following alternative explanation that focuses on the dynamic coupling of the convection with the frictional boundary layer. First, we note that the weaker downdrafts in CBLAST $_{68}$ and CBLAST $_{54}$ are associated with weaker updraft activity. This may be expected because a prominent downdraft formation mechanism is the evaporation of precipitation that is formed in the updrafts and falls into unsaturated air. Both the smaller value of $C_{D}$ and $C_{K}$ in CBLAST may contribute to weaker updrafts. Ascent out of the boundary layer forced by frictional convergence, either underneath the eyewall or underneath the vorticity anomaly associated with the tilt of the outer vortex (RMN10, their Fig. 17), can be expected to decrease for decreasing $C_{D}$. For example, in simple Ekman pumping theory the vertical motion at the top of the boundary layer is proportional to $\sqrt{C_{D}}$ (e.g., Holton,
2004, his Eq. 5.38). The smaller value of $C_{K}$ in CBLAST decreases the rate by which heat is extracted from the ocean surface. These reduced surface fluxes destabilise the boundary layer in CBLAST to a lesser extent than in RMN. Less vigorous updrafts can be expected to occur in such a more stable stratification. Our foregoing argument is supported by the results of the CBLAST and RMN no-shear experiments (not shown). Without vertical shear, the activity of transient rain bands and the associated localized downward flux of low $\theta_{\mathrm{e}}$ into the inflow layer in CBLAST are less pronounced than in the no-shear RMN experiment also.

In all experiments, vertical wind shear increases the frequency of moderate to strong downdrafts. It is clear that CBLAST has the lowest frequency of strong downdrafts and that ICE has the highest frequency. The downdraft activity as seen in the CFADs is consistent with the magnitude of the inflow layer $\theta_{\mathrm{e}}$ depression outside of the eyewall (cf. Fig. 8). Therefore, the examination of the CFADs provides additional support for the hypothesis that the distinct depression of the inflow layer $\theta_{\mathrm{e}}$ values are caused by downdrafts induced by the imposed vertical wind shear.

\section{A closer look at the $\mathrm{ICE}_{68}$ experiment}

The weakening of the $\mathrm{TC}$ in $\mathrm{ICE}_{68}$ is much more pronounced than in the comparable warm-rain experiments $\mathrm{RMN}_{68}$ and CBLAST $_{68}$. Nevertheless, in the previous sections it has been shown that the general structural changes in the first $20 \mathrm{~h}$ are very similar in all experiments. We now take a closer look at $\mathrm{ICE}_{68}$ to further confirm that the intensity evolution in this case is consistent with the shear-induced thermodynamic modification of the inflow layer. First, the inflow layer $\theta_{\mathrm{e}}$ distribution is examined during periods of distinct intensity changes. Then, we will provide evidence based on axisymmetric spindown theory that the rapid weakening in the first $20 \mathrm{~h}$ is consistent with the spindown of the TC vortex due to divergence above the inflow layer associated with a significant decrease of the inner-core convective mass flux. 

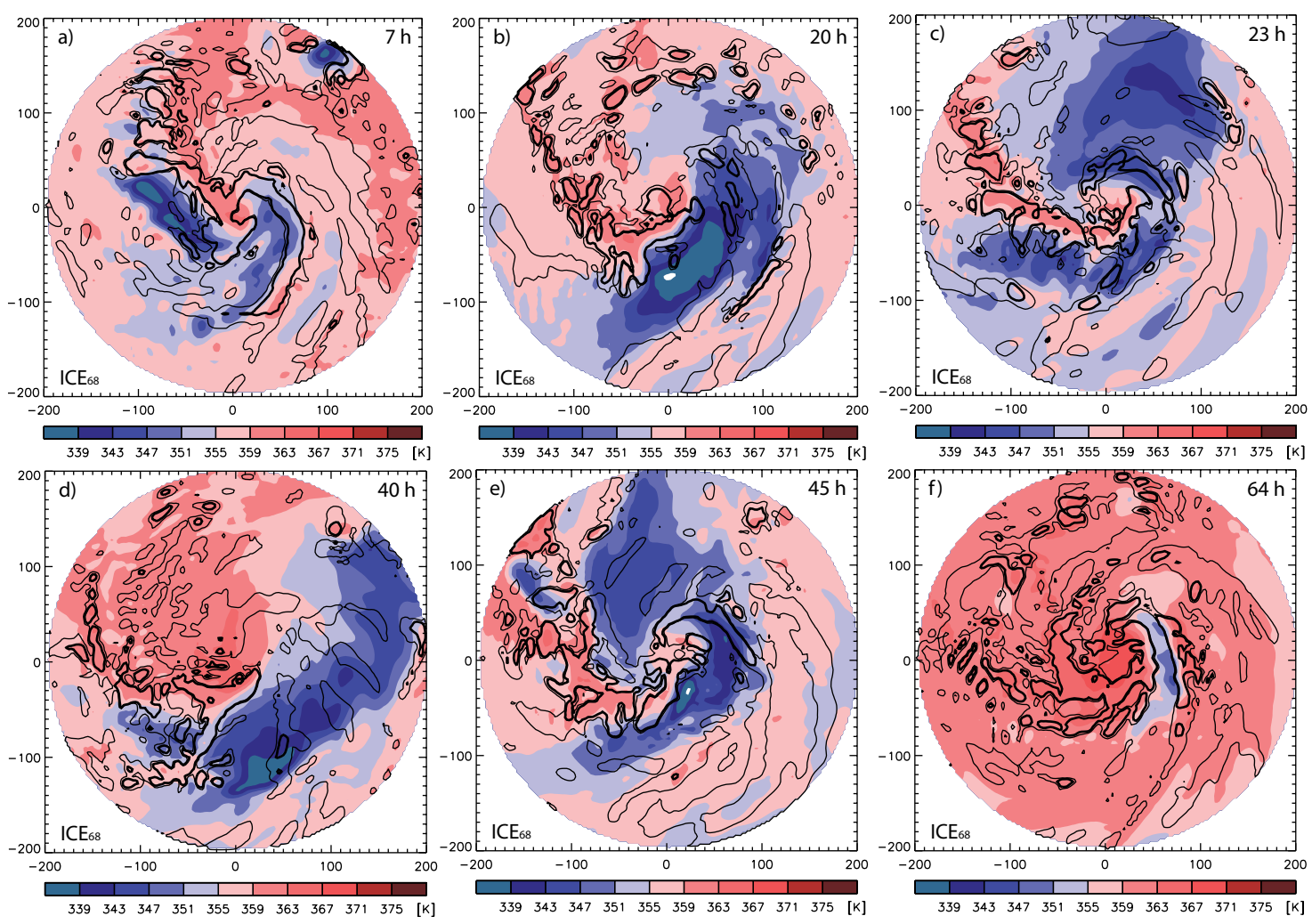

Fig. 11. As Fig. 7, but for $\mathrm{ICE}_{68}$ at (a) $7 \mathrm{~h}$, (b) $20 \mathrm{~h}$, (c) $23 \mathrm{~h}$, (d) $40 \mathrm{~h}$, (e) $45 \mathrm{~h}$, and (f) $64 \mathrm{~h}$.

\subsection{Intensity evolution and inflow layer $\theta_{\mathrm{e}}$}

It has been shown in Sect. 3.6 (Fig. 7e) that the rapid weakening is associated with a significant depression of inflow layer $\theta_{\mathrm{e}}$ soon after shear is imposed. Here we emphasize that a significant part of the depressed- $\theta_{\mathrm{e}}$ air apparently intrudes from the downshear quadrant into the inner-core updrafts on a direct pathway (Fig. 11a, at $7 \mathrm{~h}$ ). Over half of the area underneath the inner-core updrafts at this time has $\theta_{\mathrm{e}}$ values lower than $355 \mathrm{~K}$, as compared to approx. $363 \mathrm{~K}$ before shear is imposed (not shown). Along with this significant frustration of the $\theta_{\mathrm{e}}$ values in the inner-core region the vertical mass flux at midlevels $(5 \mathrm{~km})$ decreases in the first $10 \mathrm{~h}$ by $60 \%$ from $50 \times 10^{6} \mathrm{~kg} \mathrm{~s}^{-1}$ to approx. $20 \times 10^{6} \mathrm{~kg} \mathrm{~s}^{-1}$ (not shown).

We consider now the two distinct, short-lived, local intensity maxima at $22 \mathrm{~h}$ and $44 \mathrm{~h}$, prominent features of the intensity evolution in $\mathrm{ICE}_{68}$ (Fig. 2b, the maxima are found at $64 \mathrm{~h}$ and $86 \mathrm{~h}$ because this figure includes the $42 \mathrm{~h}$ spinup period). Both periods of temporary re-intensification are preceded by relatively high inflow layer $\theta_{\mathrm{e}}$ values in the downshear to downshear-right quadrants while the very pronounced $\theta_{\mathrm{e}}$ depression at these times is mostly confined to the upshear semicircle (at $20 \mathrm{~h}$ (Fig. 11b) and at $40 \mathrm{~h}$ (Fig. 11d)). Due to the distinct wave-number 1 inflow asymmetry discussed previously (Fig. 8), it can be expected that, at these times, the eyewall updrafts are fed to a large extent by the relatively high- $\theta_{\mathrm{e}}$ air in the downshear semicircle. Eyewall $\theta_{\mathrm{e}}$ values may then temporarily increase and the TC may intensify. The temporary intensification ensues until the very low $\theta_{\mathrm{e}}$ air from the upshear semicircle wraps around the centre and limits considerably the supply of relatively high $\theta_{\mathrm{e}}$ (exemplified at $23 \mathrm{~h}$ (Fig. 11c) and at $45 \mathrm{~h}$ (Fig. 11e)).

At the end of our experiment a robust re-intensification is indicated in the intensity time series (Fig. 2b). As in the experiments examined in RMN10, this final re-intensification is associated with an almost complete recovery of the inflow layer $\theta_{\mathrm{e}}$ (Fig. 11f).

As indicated in Fig. 11b-e, low- $\theta_{\mathrm{e}}$ air in the inflow layer may wrap around the centre into the downshear-right quadrant. This low- $\theta_{\mathrm{e}}$ air tends to suppress the convection of the SBC. Furthermore, after $20 \mathrm{~h}$ in $\mathrm{ICE}_{68}$, the vortex tilt is no longer in the approximate left-of-shear equilibrium direction, but starts to precess. In accordance with this precession, the vorticity anomaly associated with the tilt of the outer vortex then starts to move also and thus no longer provides persistent forcing for the SBC in the downshear-right quadrant. It is interesting to note that a narrow band of convection preferentially occurs in the downshear quadrant at these later times (Fig. 11b-e). Frequently, one observes significant downward flux of low $\theta_{\mathrm{e}}$ associated with this remnant SBC (not shown). 


\subsection{A timescale for vortex spindown}

Within the simplified framework of an idealised Carnot cycle, and based on the idea that environmental vertical wind shear leads to the frustration of such a thermodynamic cycle, Tang and Emanuel (2010) have derived a theory for the storm's reduced steady-state intensity. Such a theory, however, does not provide information about the temporal evolution of storm intensity, i.e. how rapidly a TC weakens. A well-known concept for the weakening of a TC is the axisymmetric, frictionally-induced spindown of the TC vortex. Frictional convergence forces ascent out of the inflow layer. When the vertical convective mass flux is smaller than the frictionally forced vertical mass flux, some of the air has to diverge above the inflow layer due to mass continuity (Willoughby, 1979). Under the assumption that air parcels approximately conserve their angular momentum above the frictional inflow layer, this divergence implies a decay of the tangential winds, i.e. the TC weakens.

A timescale for the frictional spindown of an axisymmetric vortex without convective mass flux has been derived by Elliassen and Lystad (1977). Their model assumes a neutral stratification above the layer of strong frictional inflow which is a reasonable assumption in the inner core of a TC because isosurfaces of $\theta_{\mathrm{e}}$ and angular momentum are approximately congruent in this region. Montgomery et al. (2001) have verified by numerical experiments that the theory gives reasonable results for the spindown of TC-like vortices, even beyond the formal validity of the theory. The half-time $t_{\text {half }}$, i.e. the time that is required to reduce the initial tangential velocity $v$ by a factor of 2, is given by Elliassen and Lystad's theory as

$t_{\text {half }}=\frac{H-h}{\chi^{2} C_{D} v}$.

Here, $H$ is the fluid depth, $h$ the height of the boundary layer, and $\chi$ a reduction factor defined by Elliassen and Lystad as the ratio of the tangential winds at the top of the surface layer to the tangential winds at the top of the Ekman layer. Estimating $H-h=10^{4} \mathrm{~m}, \chi=0.8$, and using $C_{D}=3.8 \times 10^{-3}$ from Deacon's formula with $v=68 \mathrm{~m} \mathrm{~s}^{-1}$ yields a half-time $t_{\text {half }}=16.8 \mathrm{~h}$.

The weakening of the TC in $\mathrm{ICE}_{68}$ is remarkable: intensity decreases from $68 \mathrm{~m} \mathrm{~s}^{-1}$ at $42 \mathrm{~h}$ to $33 \mathrm{~m} \mathrm{~s}^{-1}$ at $68 \mathrm{~h}$ (Fig. 2b), corresponding to a weakening rate of $35 \mathrm{~m} \mathrm{~s}^{-1}$ in $26 \mathrm{~h}$; or a half-time of approx. $25 \mathrm{~h}$. This value is consistent with a partial shutdown of the convective mass flux. As noted above, concurrently with the frustration of the inflow layer $\theta_{\mathrm{e}}$ and the rapid weakening, the vertical mass flux at $5 \mathrm{~km}$ height decreases by approx. $60 \%$. Consequently, it can be argued that the significant reduction of the convective vertical mass flux by the severe depression of the inflow layer $\theta_{\mathrm{e}}$ values broadly governs the rapid weakening in $\mathrm{ICE}_{68}$.

\section{Conclusions}

In the light of a new framework developed in a recent study (RMN10), the current study examines further the evolution of TCs exposed to environmental vertical wind shear. The new framework has been developed on the basis of idealized numerical experiments for which we have deliberately chosen a simple set of physical parameterisations to minimize the complexity of the problem. In the current study, we assess the robustness of our previous results in a more realistic and representative experimental setup by surveying and diagnosing five additional numerical experiments.

The modifications of the experimental setup focus on parameters that are important for structure change and intensity evolution of numerically simulated TCs and the storms' evolution in vertical wind shear, discussed in some detail in the Introduction in Sect. 1.2. Specifically, in comparison to RMN10, we here consider weaker, but still mature TCs; employ values for the exchange coefficients of surface heat and momentum fluxes that are in better agreement with recent observational results; and complement our previous warmrain experiments with experiments that employ an ice microphysics scheme.

Several environmental factors, such as the vertical profiles of moist entropy and the environmental winds, likely play a role for the evolution of TCs in vertical wind shear also. Furthermore, the motion vector of the TCs in all of our experiments is approx. $30^{\circ}-45^{\circ}$ left of the shear vector (not shown) while TCs in the real atmosphere generally feature a more pronounced and more variable angle. The role of the environmental factors and of storm motion constitutes an important topic for future research. It is hoped that the framework presented in RMN10, Riemer and Montgomery (2011) and in the current study provides a helpful conceptual basis for such research.

The ability of TC-like vortices to withstand the differential advection by vertical wind shear and to eventually re-align depends on the radial profile of the swirling winds (discussed in detail in Sect. 3.1). Some differences in this radial structure arise in the individual experiments before vertical wind shear is imposed. The most notable differences are found in the experiment with ice microphysics in which the TC, inter alia, features a radius of maximum winds that is approx. $25 \%$ smaller than in the warm-rain experiments. Differences in the radial vortex structure complicate the attribution of differences in the TC evolution to the inclusion of ice microphysics alone.

The differences in the vortex structure notwithstanding, in all experiments the TCs exhibit the same general structural changes when exposed to vertical wind shear. The structural changes are consistent with RMN10's new framework and comprise a vortex tilt predominantly to the downshear-left for 20-24 h, the formation of a pronounced wave-number 1 convective asymmetry outside of the eyewall (reminiscent of a stationary band complex), and downward flux of low- $\theta_{\mathrm{e}}$ air 
into the inflow layer in the downshear-left semicircle. A significant depression of the inflow layer $\theta_{\mathrm{e}}$ values $(\mathcal{O}(10 \mathrm{~K}))$ occurs in all experiments.

Furthermore, for all experiments the potential for a nonlinear, Kelvin-Helmholtz type instability in the inner core of the tilted TC-vortex is diagnosed. Such an instability would contribute to enhanced dissipation of kinetic energy and may modify the flux of low- $\theta_{\mathrm{e}}$ air into the inflow layer. However, a quantitative analysis of the mixing process associated with the potential instability is deferred to future work.

Two additional diagnostics are considered in the current study that have not been considered in RMN10, namely the $\theta_{\mathrm{e}}$ values underneath the asymmetric inner-core updrafts, and so-called contoured frequency by altitude diagrams to examine the general characteristics of vertical motion. The magnitude of the $\theta_{\mathrm{e}}$ depression underneath the asymmetric innercore updrafts early in the respective shear experiment correlates well with the ensuing weakening of the TC, supporting the idea that it is the depression of the inflow layer $\theta_{\mathrm{e}}$ that governs the intensity evolution to first order. For all experiments, vertical wind shear increases the frequency of strong downdrafts as compared to the no-shear experiments. The magnitude of the $\theta_{\mathrm{e}}$ depression outside of the eyewall is consistent with the frequency of strong downdrafts, supporting the idea that it is the shear-induced downdrafts that flush low$\theta_{\mathrm{e}}$ air into the inflow layer. Consequently, both additional diagnostics corroborate RMN10's framework.

In one of our experiments employing ice microphysics, a very rapid weakening of the TC occurs. This intensity evolution is distinct from the intensity evolution in our warm-rain experiments. Therefore, a question may naturally arise whether ice microphysics, as represented in the RAMS model, introduce an important mechanism for intensity change that is not considered in RMN10's framework. As noted above, the structural changes caused by the vertical wind shear in the experiment with ice microphysics are very similar to those in the warm-rain experiments. In our suite of experiments, the general downdraft activity, the flux of low$\theta_{\mathrm{e}}$ air into the inflow layer, and the $\theta_{\mathrm{e}}$ depression underneath the inner-core updrafts are all the most pronounced in the ice experiment. The inner-core $\theta_{\mathrm{e}}$ depression that accompanies the initial rapid weakening is more than twice as high as that in the comparable experiments employing warm-rain microphysics (6-7 K as compared to 2-3 K). Axisymmetric spindown theory (Elliassen and Lystad, 1977; Montgomery et al., 2001) further supports the hypothesis that the rapid initial weakening is due to a substantial decrease of the convective mass flux associated with the significant depression of the inflow layer $\theta_{\mathrm{e}}$ by shear-induced downdrafts. In addition, we have documented a close relationship between the evolution of the inflow layer $\theta_{\mathrm{e}}$ values and two transient intensity maxima that occur at $22 \mathrm{~h}$ and $44 \mathrm{~h}$ after vertical wind shear is imposed. The final re-intensification of the TC in this experiment, as in RMN10, is associated with a near-complete replenishment of the inflow layer $\theta_{\mathrm{e}}$ values also.
Based on the evidence presented here, we conclude that RMN10's framework for the intensity modification of TCs in vertical wind shear is robust to the presented changes in the setup of the numerical experiment. We advocate further study and tests of this framework with case studies and real atmospheric data.

Acknowledgements. John Molinari's comments during the review process of RMN10 (available online through the ACPD website at http://www.atmos-chem-phys-discuss.net/9/10711/2009/ acpd-9-10711-2009.html) were very helpful for the current study. The thoughtful comments by Roger Smith and an anonymous reviewer have helped to improve the presentation of our results. Most of this research was performed while the first author held a National Research Council Research Associateship Award at the Naval Postgraduate School in Monterey, CA, USA. This work was supported by NSF grant ATM-0715426, the ONR grant N0001411WX20095 and NOAA's Hurricane Research Division.

Edited by: T. J. Dunkerton

\section{References}

Abarbanel, H. D. I., Holm, D. D., Marsden, J. E., and Ratiu, T.: Nonlinear stability of incompressible stratified flow, Philos. T. Roy. Soc. A, 318, 349-409, 1986.

Bell, M. M., Montgomery, M. T., and Emanuel, K. A.: Air-sea enthalpy and momentum exchange at major hurricane wind speeds observed during CBLAST, J. Atmos. Sci., 69, 3197-3222, 2012.

Bender, M. A.: The effect of relative flow on the asymmetric structure in the interior of hurricanes, J. Atmos. Sci., 54, 703-724, 1997.

Black, P. G., D’Asaro, E. A., Drennan, W. M., French, J. R., Niiler, P. P., Sanford, T. B., Terrill, E. J., Walsh, E. J., and Zhang, J. A.: Air-sea exchange in hurricanes: synthesis of observations from the Coupled Boundary Layer Air-Sea Transfer experiment, B. Am. Meteorol. Soc., 88, 357-374, 2007.

Braun, S. A. and Tao, W. K.: Sensitivity of high-resolution simulations of Hurricane Bob (1991) to planetary boundary layer parameterizations, Mon. Weather Rev., 128, 3941-3961, 2000.

Bui, H. H., Smith, R. K., Montgomery, M. T., and Peng, J.: Balanced and unbalanced aspects of tropical-cyclone intensification, Q. J. Roy. Meteor. Soc., 135, 715-1731, 2009.

Cotton, W. R., Pielke Sr., R. A., Walko, R. L., Liston, G. E., Tremback, C. J., Jiang, H., McAnelly, R. L., Harrington, J. Y., Nicholls, M. E., Carrio, G. G., and McFadden, J. P.: RAMS 2001: current status and future directions, Meteorol. Atmos. Phys., 82, 5-29, 2003.

DeMaria, M.: The effect of vertical shear on tropical cyclone intensity change, J. Atmos. Sci., 53, 2076-2088, 1996.

Eliassen, A. and Lystad, M.: On the Ekman layer in a circular vortex: a numerical and theoretical study, Geophys. Norv., 31, 1-16, 1977.

Emanuel, K. A.: Atmospheric convection, Oxford University Press, New York, USA, 172 pp., 1994.

Fovell, R. G., Corbosiero, K. L., and Kuo, H.-C.: Cloud microphysics impact on hurricane track as revealed in idealized experiments, J. Atmos. Sci., 66, 1764-1778, 2009. 
Frank, W. M. and Ritchie, E. A.: Effects of vertical wind shear on the intensity and structure of numerically simulated hurricanes, Mon. Weather Rev., 129, 2249-2269, 2001.

Hill, G. E.: Factors controlling the size and spacing of cumulus clouds as revealed by numerical experiments, J. Atmos. Sci., 31, 646-673, 1974.

Holton, J.: An introduction to dynamic meteorology, AcademicPress, London, 132 pp., 2004.

Jones, S. C.: The evolution of vortices in vertical shear. I: Initially barotropic vortices, Q. J. Roy. Meteor. Soc., 121, 821-851, 1995.

Kessler, E.: On the distribution and continuity of water substance in atmospheric circulations, Meteor. Mon., 32, 84 pp., 1969.

Lilly, D. K.: On the numerical simulation of buoyant convection, Tellus, 14, 148-172, 1962.

Lord, S. J., Willoughby, H. E., and Piotrowicz, J. M.: Role of a parameterized ice-phase microphysics in an axisymmetric, nonhydrostatic tropical cyclone model, J. Atmos. Sci., 41, 2836-2848, 1984.

Meyers, M., Walko, R., Harrington, J., and Cotton, W.: New RAMS cloud microphysics prameterization. Part II: The two-moment scheme., Atmos. Res., 45, 3-39, 1997.

Montgomery, M. T., Snell, H. D., and Yang, Z.: Axisymmetric spindown dynamics of hurricane-like vortices, J. Atmos. Sci., 58, 421-435, 2001.

Montgomery, M. T., Smith, R. K., and Nguyen, S. V.: Sensitivity of tropical cyclone models to the surface drag coefficient, Q. J. Roy. Meteor. Soc., 136, 1945-1953, 2010.

Pielke, R. A., Cotton, W. R., Walko, R. L., Tremback, C. J., Lyons, W. A., Grasso, L. D., Nicholls, M. E., Moran, M. D., Wesley, D. A., Lee, T. J., and Copeland, J. H.: A comprehensive meteorological modeling system - RAMS, Meteorol. Atmos. Phys., 49, 69-91, 1992.

Reasor, P. D., Montgomery, M. T., and Grasso, L. D.: A new look at the problem of tropical cyclones in vertical shear flow: vortex resiliency, J. Atmos. Sci., 61, 3-22, 2004.

Riemer, M. and Montgomery, M. T.: Simple kinematic models for the environmental interaction of tropical cyclones in vertical wind shear, Atmos. Chem. Phys., 11, 9395-9414, doi:10.5194/acp-11-9395-2011, 2011.

Riemer, M., Montgomery, M. T., and Nicholls, M. E.: A new paradigm for intensity modification of tropical cyclones: thermodynamic impact of vertical wind shear on the inflow layer, Atmos. Chem. Phys., 10, 3163-3188, doi:10.5194/acp-10-31632010, 2010.

Schecter, D. A. and Montgomery, M. T.: On the symmetrization rate of an intense geophysical vortex, Dynam. Atmos. Oceans, 37, 55-88, 2003.

Smagorinsky, J.: General circulation experiments with the primitive equations, Mon. Weather Rev., 91, 99-164, 1963.
Smith, R. K. and Thomsen, G. L.: Dependence of tropical-cyclone intensification on the boundary-layer representation in a numerical model, Q. J. Roy. Meteor. Soc., 136, 1671-1685, 2010.

Smith, R. K., Montgomery, M. T., and Thomsen, G.: Sensitivity of tropical cyclone models to the surface drag coefficient in different boundary-layer schemes, Q. J. Roy. Meteor. Soc., in review, 2012.

Srivastava, R. C.: A model of intense downdrafts driven by the melting and evaporation of precipitation, J. Atmos. Sci., 44, 17521774, 1987.

Tang, B. and Emanuel, K. A.: Midlevel ventilation's constraint on tropical cyclone intensity, J. Atmos. Sci., 67, 1817-1830, 2010.

Tripoli, G. J. and Cotton, W. R.: The use of ice-liquid water potential temperature as a thermodynamic variable in deep atmospheric models, Mon. Weather Rev., 109, 1094-1102, 1981.

Verlinde, J., Flatau, P., and Cotton, W.: Analytical solutions to the collection growth equation: comparison with approximate methods and application to cloud microphysics parameterization schemes, J. Atmos. Sci., 47, 2871-2880, 1990.

Walko, R., Cotton, W., Harrington, J., and Meyers, M.: New RAMS cloud microphysics parameterization. Part I: The single-moment scheme, Atmos. Res., 38, 29-62, 1995.

Wang, J.: Does the vertical wind shear profile matter to tropical cyclone intensity change?, 30th Conf. on Hurricanes and Tropical Meteorology, Ponte Verde, FL, Amer. Meteor. Soc., https://ams.confex.com/ams/30Hurricane/flvgateway. cgi/id/20692?recordingid=20692, 2012.

Willoughby, H. E.: Forced secondary circulations in hurricanes, J. Geophys. Res., 84, 3173-3183, 1979.

Willoughby, H. E., Marks, F. D., and Feinberg, R. J.: Stationary and moving convective bands in hurricanes, J. Atmos. Sci., 41, 31893211, 1984.

Wong, M. L. M. and Chan, J. C. L.: Tropical cyclone intensity in vertical wind shear, J. Atmos. Sci., 61, 1859-1876, 2004.

Yuter, S. E. and Houze, R. A.: Three-dimensional kinematic and microphysical evolution of Florida cumulonimbus. Part II: Frequency distributions of vertical velocity, reflectivity, and differential reflectivity, Mon. Weather Rev., 123, 1941-1963, 1995.

Zeng, Z., Wang, Y., and Chen, L.: A statistical analysis of vertical shear effect on tropical cyclone intensity change in the North Atlantic, Geophys. Res. Lett., 37, L02802, doi:10.1029/2009GL041788, 2010.

Zhang, J. A., Black, P. G., French, J. R., and Drennan, W. M.: First direct measurements of enthalpy flux in the hurricane boundary layer: the CBLAST results, Geophys. Res. Lett., 35, L14813, doi:10.1029/2008GL034374, 2008. 